\title{
ON DIFFUSE INTERFACE MODELING AND SIMULATION OF SURFACTANTS IN TWO-PHASE FLUID FLOW
}

\author{
STEFAN ENGBLOM, MINH DO-QUANG, GUSTAV AMBERG, \\ AND ANNA-KARIN TORNBERG
}

\begin{abstract}
An existing phase-field model of two immiscible fluids with a single soluble surfactant present is discussed in detail. We analyze the well-posedness of the model and provide strong evidence that it is mathematically ill-posed for a large set of physically relevant parameters. As a consequence, critical modifications to the model are suggested that substantially increase the domain of validity. Carefully designed numerical simulations offer informative demonstrations as to the sharpness of our theoretical results and the qualities of the physical model. A fully coupled hydrodynamic test-case demonstrates the potential to capture also non-trivial effects on the overall flow.
\end{abstract}

\section{INTRODUCTION}

The presence of surface active substances may greatly affect the physical properties of fluid mixtures. Indeed, these effects are used critically in many important applications in everyday life; detergents and oil-water emulsions in food are two immediate examples. The fact that surfactants lower the surface tension is exploited in both of these cases: detergents make the water more "wet", and an emulsifying agent stabilizes an emulsion by preventing small droplets to coalesce.

For humans, probably the most critical everyday usage of surfactants is made in the alveoli in the lung, where pulmonary surfactant, amongst other things, prevents lung collapse at the end of expiration $[22,26]$.

An interesting and very striking realization in vivo was reported recently in [32] where chemotaxis was implemented for small droplets of fluid in a bulk solution, physically contained in a maze. The net transport at the millimeter-scale, and the subsequent solution to the maze, was achieved by a clever usage of surfactant and a pre-existing pH-gradient. Quite likely, such a constructive set-up could find applications in lab-on-a-chip manufacturing.

Consisting of hydrophobic "heads" and hydrophilic "tails", surfactant molecules have a strong preference to occupy sites at the water-fluid or water-gas interfaces. Below the critical micelle concentration (CMC), surfactants therefore adsorb efficiently to the interfaces where their physical effects become prominent. Above the CMC, additionally, spontaneous formation of stable groups of surfactants micelles — occurs in the bulk solution [38].

Date: October 5, 2012.

2010 Mathematics Subject Classification. Primary: 76T30; Secondary: 65M60, 65Z05.

Key words and phrases. Phase-field model, Cahn-Hilliard equation, surface active agent, Ginzburg-Landau free energy, well-posedness.

Corresponding author: S. Engblom, telephone +46-18-471 27 54, fax +46-18-51 1925. 
Given the 'thermodynamical' signature of surfactants; that is, the diffusionlimited flow into the interfaces and the spontaneous creation of highly regular micelles from an unordered state in the bulk, modeling through some kind of system's energy assumption is a tempting approach.

Ariel, Diamant, and Andelman [1,9] have successfully postulated free-energy terms with theoretically convincing properties. Their approach is inherently sharp in that they set up equations for the interface, the sub-surface region, and the bulk. A later work [38] shows that this methodology can be extended to include also the region above the CMC. A great feature with this type of modeling is the fact that all properties of the model result from a single postulated entity; the system's free energy.

Given the multitude of scales present and the complex coupling to hydrodynamics, numerical experiments become important as tools to gain a better understanding and a fuller physical insight.

In a so-called sharp interface method, the interface is considered to be infinitesimally thin, and its exact location is represented either explicitly (front-tracking with e.g. Lagrangian markers), or implicitly (e.g. level-set [55] and volume of fluid (VOF) methods [28]). The evolution of the surfactant concentration on each interface can be described by a partial differential equation on this time-dependent manifold [47]. Techniques for solving this PDE and include insoluble surfactants in multiphase flow simulations have been developed based on several different interface representation techniques [3, 28, 31, 33, 42, 55].

When the surfactants are soluble also in the bulk, source terms due to adsorption and desorption terms enter this PDE, and this must be coupled to a PDE for the bulk concentration of surfactants with appropriate boundary conditions for the surfactant flux. See e.g. Eggleton and Stebe [13] for an early reference. To simplify matters for simulations, it is in this reference assumed that the adsorption and desorption from the bulk is diffusion dominated and that the bulk surfactant concentration is spatially constant. Work to consider the full problem has started only recently and is currently an active area of research [5, 30, 39, 50, 57].

Given a thermodynamic description as a postulated free energy of the system, including surface effects introduced via gradient energy terms [34, 35, 36, 44, 51, 52], it is attractive to represent the interface as a rapid but continuous transition in a concentration variable. This is the essence of phase-field or diffuse interface modeling. Without the need to explicitly track the interface, even complicated topological transformations can be studied, and the coupling to the full hydrodynamic flow is fairly straightforward [27].

However, this has only recently begun to be explored for surfactant laden interfaces. In [50], a phase-field method was used to represent the interfaces. The surfactant treatment was introduced in a manner quite natural to a sharp interface method, and was not included in a thermodynamical derivation of a diffuse interface method for the full problem. In [44], and with some additions, in [36], a seemingly natural diffuse interface model for surfactant laden liquid-liquid mixtures is presented, and solved with lattice Boltzmann methods. The model is verified against classical results, and some cases of convection dominated flow, such as a deforming droplet in a shear flow are presented. This formulation is promising from a computational and physical point of view, but it is rather complex and its mathematical properties have not been investigated. We will show below that this model can 
in fact be expected to be mathematically ill-posed under certain conditions. The purpose of the present paper is to clarify the conditions under which the model is ill-posed, and also to present variants that behave better.

In Section 3 we thus present the analysis of the model, and we also derive three alternatives in the form of modifications to the original model. The properties of these alternatives are investigated in Section 4 using a highly accurate one-dimensional numerical scheme, and in a more qualitative sense in two spatial dimensions in Section 5. An outlook with conclusions is finally found in Section 6.

\section{Diffuse interface MODEL FOR SURFACTANT FLOW}

In this section we shall discuss our 'baseline' phase-field model for multiphase flow incorporating surfactants; - later we shall have reasons to alter the actual model by incorporating new terms. Our starting point is a model originally presented in [44], but incorporating the work also of others.

Specifically, a diffuse interface model for surfactant-controlled emulsification was proposed in [52] and a related model for oil-water-detergent mixtures appeared in [34]. A model with more easily understood interface adsorption properties was thus proposed in [44], incorporating free energy terms developed previously for a sharp model [10], but drawing also on some earlier work [35]. For clarity we take a nondimensional version as our baseline model (see Appendix A for the precise relation to the original model in [44]).

The degrees of freedom are the phase-field variable $\phi \in[-1,1]$, the surfactant volume fraction $\psi \in[0,1]$, and the fluid velocity field $\mathbf{u}$. The governing equations for $\phi$ and $\psi$ take the form of Cahn-Hilliard-type equations [27],

$$
\begin{aligned}
& \frac{\partial \phi}{\partial t}+\nabla \cdot(\phi \mathbf{u})=\frac{1}{\mathrm{Pe}_{\phi}} \nabla \cdot M_{\phi} \nabla \mu_{\phi}=\frac{1}{\mathrm{Pe}_{\phi}} \Delta \mu_{\phi}, \\
& \frac{\partial \psi}{\partial t}+\nabla \cdot(\psi \mathbf{u})=\frac{1}{\mathrm{Pe}_{\psi}} \nabla \cdot M_{\psi} \nabla \mu_{\psi},
\end{aligned}
$$

in terms of Péclet numbers Pe, mobilities $M$, and chemical potentials $\mu$ which will be prescribed below. As indicated in (2.1) the model is somewhat simplified in that it assumes the "shallow quench" limit [41, Sect. 9], and hence that the mobility $M_{\phi}$ for $\phi$ is assumed to be constant (and in fact normalized to unity by scaling the Péclet number $\mathrm{Pe}_{\phi}$ appropriately). This simplification is quite standard and is adopted here mainly for convenience.

Eqs. (2.1)-(2.2) are coupled to the Navier-Stokes equations in the form

$$
\begin{aligned}
\nabla \cdot \mathbf{u} & =0, \\
\rho\left(\frac{\partial \mathbf{u}}{\partial t}+\mathbf{u} \cdot \nabla \mathbf{u}\right)= & -\nabla P+\frac{1}{\operatorname{Re}} \nabla \cdot\left(\rho \nu\left[\nabla \mathbf{u}+(\nabla \mathbf{u})^{T}\right]\right) \\
& -\frac{1}{\operatorname{CaCnRe}}\left(\phi \nabla \mu_{\phi}+\psi \nabla \mu_{\psi}\right)
\end{aligned}
$$

where the pressure tensor $P$ enforces the incompressibility condition (2.3), and where Re is the Reynolds number, Ca the Capillary number, and where, respectively, $\rho$ and $\nu$ denote the fluid's density and kinematic viscosity. In the general case, $\rho$ and $\nu$ may depend on $\phi$ as well as on other state variables, requiring that additional effects be taken into account [11]. For our present purposes and for simplicity they are assumed to be constants. 
2.1. The Ginzburg-Landau free energy. Recall that in diffuse models of binary fluids in equilibrium, the phase-field variable $\phi$ typically describes a planar interface at the origin of the form

$$
\phi(x)=\tanh (x / \mathrm{Cn}),
$$

where $\mathrm{Cn}$ is the Cahn number expressing the ratio between the interface width and the characteristic length scale.

In (2.1)-(2.2) the chemical potentials are derived from a Ginzburg-Landau free energy functional,

$$
\int_{\Omega} F d x \equiv \int_{\Omega} F_{\phi}+F_{\psi}+F_{1}+F_{\text {ex }} d x
$$

The classical constant mobility Cahn-Hilliard potential [41, Sect. 4] is given by

$$
F_{\phi}=-\frac{\phi^{2}}{2}+\frac{\phi^{4}}{4}+\frac{\mathrm{Cn}^{2}}{4}(\nabla \phi)^{2}
$$

where we note that the polynomial part is a "double well" potential, $\left[\left(\phi^{2}-1\right)^{2}-1\right] / 4$, and hence expresses a preference to pure (non-mixed) phases $\phi= \pm 1$. Also, the explicit penalty term for steep gradients causes any interfaces to become diffused.

For $\psi$, a logarithmic free energy is rather preferred as this provides for certain analytical properties such as possessing an isotherm relation as outlined in Section 4.2 ,

$$
F_{\psi}=\operatorname{Pi}[\psi \log \psi+(1-\psi) \log (1-\psi)] .
$$

The temperature-dependent constant Pi (cf. (A.11)) takes the role of a diffusion coefficient for $\psi$ and $F_{\psi}$ is thus the energy potential governing the entropy decrease of mixing the surfactant with the bulk phase. We shall later find reasons to modify (2.8) since it, unlike (2.7) does not contain a square gradient term. Note that a logarithmic free energy is to be combined with a degenerate mobility which vanishes at the extreme points $\psi \in\{0,1\}$ [41, Sect. 4.2]. The usual choice is simply

$$
M_{\psi}=\psi(1-\psi)
$$

which will also be used here. A point in favor of formulating the Cahn-Hilliard equation using this kind of mobility is that, unlike the constant mobility case, it can rigorously be shown to produce solutions $0 \leq \psi \leq 1$ [41, Sect. 4.2].

Surfactant molecules tend to move to a point and orient themselves in such a way that they are at ease. Owing to their nature this generally means at the interface between fluids, with the hydrophilic part pointing towards a fluid of the system and the hydrophobic part pointing away from this fluid. In the current model, $F_{1}$ is the surface energy potential accounting for this adsorption,

$$
F_{1}=-\frac{\mathrm{Cn}^{2}}{4} \psi(\nabla \phi)^{2} \text {. }
$$

This particular form is inspired by the corresponding term in the sharp interface model of $[9,10]$. The square gradient acts as a diffuse version of the sharp interface indicator function and can rigorously be interpreted as a nascent Dirac delta function as outlined in Section 3.2.2 below. 
Finally, to conclude the specification of the free energy, the term $F_{\text {ex }}$ takes the form

$$
F_{\text {ex }}=\frac{1}{4 \mathrm{Ex}} \psi \phi^{2}
$$

and penalizes free surfactant in the respective phases. Similarly to $F_{\psi}, F_{\text {ex }}$ is an enthalpic term measuring the cost of free surfactant. However, due to the factor $\phi^{2}, F_{\text {ex }}$ is inactive at an interface where $\phi \approx 0$. Actually, (2.11) is just the simplest form among many possibilities (cf. [34, 35, 52]). To some extent, (2.10) and (2.11) are complementary: $F_{1}$ locally attracts surfactant to an existing interface while $F_{\text {ex }}$ globally counteracts the occurrence of free surfactant. Finally, and as we shall see in Section 4.1, (2.11) can also be regarded as defining the bulk solubility.

Through variational derivatives of the free energy $F$ with respect to the two variables $\phi$ and $\psi$ one now obtains the chemical potentials

$$
\begin{aligned}
& \mu_{\phi}=\frac{\delta F}{\delta \phi}=-\phi+\phi^{3}-\frac{\mathrm{Cn}^{2}}{2} \Delta \phi+\frac{\mathrm{Cn}^{2}}{2} \psi \Delta \phi+\frac{\mathrm{Cn}^{2}}{2} \nabla \psi \cdot \nabla \phi+\frac{1}{2 \mathrm{Ex}} \psi \phi, \\
& \mu_{\psi}=\frac{\delta F}{\delta \psi}=\operatorname{Pi} \log \frac{\psi}{1-\psi}-\frac{\mathrm{Cn}^{2}}{4}(\nabla \phi)^{2}+\frac{1}{4 \mathrm{Ex}} \phi^{2} .
\end{aligned}
$$

Note that, carrying out the differentiation in the right-hand side of (2.2) for the logarithmic term in (2.13), we get using (2.9) that

$$
\frac{1}{\mathrm{Pe}_{\psi}} \nabla \cdot M_{\psi} \nabla \mathrm{Pi}\left(\log \frac{\psi}{1-\psi}\right)=\frac{\mathrm{Pi}}{\mathrm{Pe}_{\psi}} \Delta \psi,
$$

provided that $0<\psi<1$.

This completes the specification of the gradient flow (2.1)-(2.2) which together with the Navier-Stokes equations (2.3)-(2.4) and suitable boundary conditions make up our basic multiphase-surfactant model. In what follows we shall initially be concerned mainly with the diffusion-controlled part (2.1)-(2.2) (with $\mathbf{u}=0$ ) and postpone computational experiments with the full hydrodynamic set of equations until Section 5. Testing (2.1) with $\mu_{\phi}$ and (2.2) with $\mu_{\psi}$, respectively, we find under natural boundary conditions that

$$
\frac{d}{d t} \int_{\Omega} F d x=-\int_{\Omega} \frac{1}{\mathrm{Pe}_{\phi}}\left\|\nabla \mu_{\phi}\right\|^{2}+\frac{M_{\psi}}{\mathrm{Pe}_{\psi}}\left\|\nabla \mu_{\psi}\right\|^{2} d x \leq 0
$$

for $M_{\psi} \geq 0$. This relation expresses the decay of the free energy and is required for thermodynamical consistency. For the full hydrodynamic set of equations we find similarly by testing (2.4) with $\mathrm{Ca} \mathrm{Cn} \operatorname{Re} \mathbf{u}$ the general energy identity

$$
\begin{aligned}
& \frac{d}{d t} \int_{\Omega} \frac{\rho \mathrm{CaCnRe}}{2}\|\mathbf{u}\|^{2}+F d x= \\
& \quad-\int_{\Omega} \frac{1}{\mathrm{Pe}_{\phi}}\left\|\nabla \mu_{\phi}\right\|^{2}+\frac{M_{\psi}}{\mathrm{Pe}_{\psi}}\left\|\nabla \mu_{\psi}\right\|^{2}+\frac{\rho \nu \mathrm{CaCn}}{2}\left\|\nabla \mathbf{u}+(\nabla \mathbf{u})^{T}\right\|^{2} d x .
\end{aligned}
$$

\section{ANALYSIS}

In this section we first show that the free-space PDE defined by (2.1)-(2.2) with $\mathbf{u}=0$ is ill-posed in the sense of frozen coefficients in a neighborhood of a sufficiently smooth steady-state solution. We then return to the baseline model (2.1)-(2.4) and modify it in order to provide for well-posedness. Three different modifications are suggested, all with differing motivations and properties. 
3.1. Ill-posedness. We thus consider the case $\mathbf{u}=0$ in (2.1)-(2.2) for a onedimensional free-space formulation. We make the quasi steady-state ansatz $\phi=$ $\phi_{\mathrm{eq}}+\delta u$ and $\psi=\psi_{\mathrm{eq}}+\delta v$, where $\phi_{\mathrm{eq}} / \psi_{\mathrm{eq}}$ are equilibrium solutions as $t \rightarrow \infty$ and where $\delta$ is a small parameter. Assuming the existence of equilibrium solutions is motivated by the fact that (2.1)-(2.2) describe a gradient flow and naturally possesses (non-unique) equilibria [48, Sect. 4]. - Fundamentally, the lack of any equilibrium would violate the second law of thermodynamics.

Assume for now that the equilibrium solutions are sufficiently regular that a linearization in $\delta$ is possible (see the discussion after (3.3) below). Upon linearizing and keeping only the principal part of the operator we get after some work the linear system of PDEs

$$
\left[\begin{array}{c}
u_{t} \\
v_{t}
\end{array}\right]=\left[\begin{array}{cc}
-\frac{\mathrm{Cn}^{2}}{2} \frac{1-\psi_{\mathrm{eq}}}{\mathrm{Pe}_{\phi}} D^{4} & \frac{\mathrm{Cn}^{2}}{2} \frac{D\left(\phi_{\mathrm{eq}}\right)}{\mathrm{Pe}_{\phi}} D^{3} \\
-\frac{\mathrm{Cn}^{2}}{2} \frac{\psi_{\mathrm{eq}}\left(1-\psi_{\mathrm{eq}}\right) D\left(\phi_{\mathrm{eq}}\right)}{\mathrm{Pe}_{\psi}} D^{3} & \frac{\mathrm{Pi}_{\phi}}{\mathrm{Pe}_{\psi}} D^{2}
\end{array}\right]\left[\begin{array}{l}
u \\
v
\end{array}\right],
$$

where $D=d / d x$. Without further ado we take the "frozen coefficient" Fourier transform of (3.1) by simply exchanging $D \rightarrow-i \omega$,

$$
\left[\begin{array}{c}
\hat{u}_{t} \\
\hat{v}_{t}
\end{array}\right]=\left[\begin{array}{cc}
-\frac{\mathrm{Cn}^{2}}{2} \frac{1-\psi_{\mathrm{eq}}}{\mathrm{Pe}_{\phi}} \omega^{4} & \frac{\mathrm{Cn}^{2}}{2} \frac{D\left(\phi_{\mathrm{eq}}\right)}{\mathrm{Pe}_{\phi}} i \omega^{3} \\
-\frac{\mathrm{Cn}^{2}}{2} \frac{\psi_{\mathrm{eq}}\left(1-\psi_{\mathrm{eq}}\right) D\left(\phi_{\mathrm{eq}}\right)}{\mathrm{Pe}_{\psi}} i \omega^{3} & -\frac{\mathrm{Pi}_{\mathrm{p}}}{\mathrm{Pe}_{\psi}} \omega^{2}
\end{array}\right]\left[\begin{array}{c}
\hat{u} \\
\hat{v}
\end{array}\right] .
$$

Call the above matrix $A$. Firstly, since the characteristic equation is real, complex eigenvalues must come in conjugate pairs. Secondly, from a quick calculation we see that $\operatorname{det} A \propto \mathrm{Pi}-\mathrm{Cn}^{2} / 2 \cdot \psi_{\mathrm{eq}}\left(D\left(\phi_{\mathrm{eq}}\right)\right)^{2}$ and hence, when the determinant is negative there is a positive real eigenvalue indicating some kind of instability. Slightly more elaborate calculations are now required to show that there is in fact an eigenvalue

$$
\lambda_{2}=\frac{1}{\mathrm{Pe}_{\psi}}\left[\frac{\mathrm{Cn}^{2}}{2} \psi_{\mathrm{eq}}\left(D\left(\phi_{\mathrm{eq}}\right)\right)^{2}-\mathrm{Pi}\right] \omega^{2}+\mathcal{O}(1)
$$

while $\lambda_{1}$ is asymptotically negative and scales as $\mathcal{O}\left(\omega^{4}\right)$. From (3.3) we see that $\lambda_{2}$ may well be positive for $\omega$ sufficiently large. If this is the case the PDE violates the Petrovskii condition which is necessary for well-posedness [19, Theorem 4.5.2].

To turn these arguments into a strict proof unfortunately requires non-trivial pointwise estimates of the equilibrium solutions. Besides the fact that there exist problems for which the frozen coefficients formulation is unstable while the variable coefficient problem is stable, the linearization argument can also be questioned. Solutions $\phi$ and $\psi$ of (2.1)-(2.2) could loose derivatives as $t \rightarrow \infty$ such that $\phi_{\text {eq }}$ and $\psi_{\text {eq }}$ are sufficiently non-smooth that the linearization argument becomes invalid. The only immediately available a priori estimates are that $\phi_{\mathrm{eq}} \in H^{1}(\Omega)$ and that $\psi_{\text {eq }} \in L^{2}(\Omega)$ (this follows directly from (2.15)). We shall not delve more into these matters here, but only remark that a linear stability analysis is well motivated from the viewpoint of physical modeling and also, that all of our numerical experiments agree very well with the analysis performed above (cf. Figure 4.1).

Using results to be developed in Section 4 we now derive some simplified conditions for the instability to occur. Let us consider it inherent in the model that both $\psi_{\text {eq }}$ and $\left(D\left(\phi_{\text {eq }}\right)\right)^{2}$ are largest at the interface (taken to be at $x=0$ ). Then $\psi_{\text {eq }} \leq$ $\psi_{\text {eq }}(0)=\psi_{0}$, the surfactant loading at the interface and, provided that the interface sharpness is independent of the surfactant loading, $\left(D\left(\phi_{\mathrm{eq}}\right)\right)^{2} \leq\left(D\left(\phi_{\mathrm{eq}}(0)\right)\right)^{2} \sim$ $1 / \mathrm{Cn}^{2}$ (see Section 4.1). Using the isotherm relation $\psi_{0} \sim \psi_{b} /\left(\psi_{c}+\psi_{b}\right)$ (again, 
see Section 4.2 below), an a priori approximate sufficient condition for instability is therefore

$$
\mathrm{Pi}<\frac{\psi_{b} / 2}{\psi_{c}+\psi_{b}}
$$

expressed in terms of the bulk value $\psi_{b}=\psi_{\mathrm{eq}}( \pm \infty)$ and the Langmuir adsorption constant $\psi_{c}$ discussed thoroughly below. Using the relation (4.16) for the latter this is equivalent to

$$
\psi_{b}>\frac{2 \mathrm{Pi}}{1-2 \mathrm{Pi}} \times \psi_{c}=\frac{2 \mathrm{Pi}}{1-2 \mathrm{Pi}} \times \exp \left(-\frac{1+1 / \mathrm{Ex}}{4 \mathrm{Pi}}\right)
$$

provided that $\mathrm{Pi}<1 / 2$. That is, sufficiently large values of $\psi_{b}$ lead to an instability near the equilibrium solution (which therefore does not exist).

This situation is somewhat remindful of results for a close relative of the CahnHilliard equation, namely the Thin film equation. This model can also be derived from a Ginzburg-Landau energy which, as in (2.15), by construction decays with time. Through a clever constructive argument, however, one can show that for a sufficiently large total mass, there is a smooth initial profile for which finitetime blowup in the form of indefinite focusing occurs (see [54] and the references therein). Unfortunately, it seems difficult to transfer these types of arguments into the current setting.

Example. In several numerical experiments in [44] using the lattice Boltzmann method, $\mathrm{Pi} \in[0.08,0.20]$ and $\mathrm{Ex} \sim 1$. To be concrete and still use actual (published) values, we take $\mathrm{Pi}=0.1227$ with $\mathrm{Ex}=1$. Then (3.5) becomes $\psi_{b}>5.526 \times 10^{-3}$ and under this condition we expect the model to be ill-posed. Since many of the experiments in [44] (see for example Fig. 1 and 2) are made in this regime, these results are misleading at the very least. One cannot help speculating that the lattice Boltzmann method used enjoy "hidden" higher order derivative terms that help increasing the stability. In Figure 3.1, p. 11 these predictions are illustrated using the numerical method developed in Appendix B. We note that similar experiments are made anew in [36] under only slightly altered conditions. Here, the parameters in the simulations appear to have been kept within the limits for well-posedness. Using this model substantially limits the physical parameter space that can be explored.

Incidentally, (3.5) disqualifies the label "numerical stabilization term" for the bulk-energy $F_{\text {ex }}$ in $(2.11)$ as used in [44, p. 4] and again in [36, p. 9167]. Increasing their variable $W$ in (A.11) (that is, "increasing" the numerical stabilization) is the same thing as decreasing Ex, thereby driving more surfactant to the interface. Assuming that the other parameters $\mathrm{Cn}$ and $\mathrm{Pi}$ are kept fixed, according to (3.5) this means that less amount of surfactant implies mathematical (as opposed to numerical) instability.

Finally, and from the perspective of physical modeling, one could in principle accept the ill-posedness as an artifact of the fact that we are dealing with an artificially diffuse interface. However, in such a case it is natural to demand at the very least that the limiting problem $\mathrm{Cn} \rightarrow 0$ is stable. For the eigenvalue $\lambda_{2}$ in (3.3) to be negative as $\mathrm{Cn} \rightarrow 0$ one clearly has to give up the natural requirement that the interface sharpness is independent of the surfactant loading. Indeed, our 
approximate bounds (3.4)-(3.5) are fully independent of $\mathrm{Cn}$ as a reflection of this assumption.

3.2. Three alternatives. In this section we seek viable alternatives to the model just analyzed. Our first modified formulation involves using the full form of the logarithmic free energy $F_{\psi}$ in (2.8). This form contains a square gradient term and leads to an additional fourth order dissipative term in the PDE for the surfactant concentration $\psi$. The downside is that the resulting model has more complicated mathematical properties and we therefore look for simpler but still reasonable alternatives. Two such suggestions are obtained by using a derivative-free diffuse interface Dirac delta function when defining $F_{1}$ in (2.10).

3.2.1. Completing the logarithmic free energy. Rather than (2.8), the complete logarithmic free energy is actually given by ([41, Sect. 4.2, Eqs. (20), (21)]; see also [14])

$$
F_{\psi}=\operatorname{Pi}[\psi \log \psi+(1-\psi) \log (1-\psi)]+\frac{\sigma}{4} \psi(1-\psi)+\frac{\mathrm{Cn}^{2}}{4}(\nabla \psi)^{2},
$$

with $\sigma$ a new parameter. Accordingly, our "Model 1" is obtained by exchanging the definition of $F_{\psi}$ in (2.8) with (3.6). While the chemical potential $\mu_{\phi}$ in (2.12) is not affected by this addition to the free energy, $\mu_{\psi}$ in (2.13) is to be replaced with

$$
\mu_{\psi}=\operatorname{Pi} \log \frac{\psi}{1-\psi}-\frac{\mathrm{Cn}^{2}}{4}(\nabla \phi)^{2}-\frac{\mathrm{Cn}^{2}}{2} \Delta \psi-\frac{\sigma}{2} \psi+\frac{1}{4 \mathrm{Ex}} \phi^{2}
$$

Evidently, one more boundary condition than before is needed since now the equation for $\psi$ is fourth order.

A possible explanation to the fact that the square gradient term is missing in the original presentation of the model in [44] is that it is also omitted in the earlier references $[9,10,35]$. In $[9,10]$ we note that the term has no meaning since the interface model there is sharp. Interestingly, the square gradient term is in fact included in the surfactant models found in $[34,51,52]$, but then for the constant mobility case. As remarked in [44] (and as will be evident in Section 4.2 below), such models do not possess a realistic adsorption isotherm.

This also seems to be the place to mention how the two order parameters $(\phi, \psi)$ are to be interpreted. Rather than a true multicomponent system as treated in [14], we are dealing with small, often very small, concentrations of surfactant. A kind of two-step approximation is therefore employed where a Cahn-Hilliard equation with degenerate mobility governs the surfactant $(\psi)$ and "non-surfactant" $(1-\psi)$ phases. In turn, the non-surfactant phase (e.g. oil and water) is treated by a classical constant mobility Cahn-Hilliard equation in the phase-field variable $\phi$. The three concentration components of the flow should therefore rightly be understood as

$$
[\psi,(1-\psi)(1+\phi) / 2,(1-\psi)(1-\phi) / 2]
$$

and are thus approximated with

$$
[\psi,(1+\phi) / 2,(1-\phi) / 2]
$$

so that the volume is only preserved up to $\mathcal{O}(\psi)$.

We conclude by offering a few comments on the lateral interaction term $-\sigma / 4 \cdot \psi^{2}$ in (3.6). This term is included also in $[9,10,36]$ where it is shown to lead to the Frumkin isotherm (see Section 4.2). Interestingly, the same term appears also in 
$[34,35,51,52]$, but here with the opposite sign. Clearly, a positive value of $\sigma$ favors clustering of surfactant since the term can be interpreted as a free energy decrease for pairs of surfactant molecules. By differentiating twice the non-gradient part of (3.6) with respect to $\psi$ we further find that

$$
\frac{d^{2}}{d \psi^{2}}\left[\operatorname{Pi}[\psi \log \psi+(1-\psi) \log (1-\psi)]+\frac{\sigma}{4} \psi(1-\psi)\right]=\frac{\mathrm{Pi}}{\psi(1-\psi)}-\frac{\sigma}{2} .
$$

Hence, for $0<\psi<1$, this part of the free energy remains convex in $\psi$ provided that we choose $\sigma \leq 8 \mathrm{Pi}$.

3.2.2. Gradient-free Dirac delta functions. We have already commented that the term $-\mathrm{Cn}^{2} / 4 \cdot \psi(\nabla \phi)^{2}$ in (2.10) results from using the square gradient as a diffuse version of the sharp interface indicator function. Indeed, for the planar interface equilibrium solution $\phi(x)=\tanh (x / \mathrm{Cn})$, we have that $(\nabla \phi)^{2}=\mathrm{Cn}^{-2} \operatorname{sech}^{4}(x / \mathrm{Cn})$. Over the real line, the quartic hyperbolic secant is a nascent Delta function in the sense that $3 /(4 \mathrm{Cn}) \operatorname{sech}^{4}(x / \mathrm{Cn}) \rightarrow \delta(x)$ (convergence in distribution in the sharp interface limit $\mathrm{Cn} \rightarrow 0)$.

Noting that the function $1-\tanh ^{2}(x / \mathrm{Cn})=\operatorname{sech}^{2}(x / \mathrm{Cn})$ also defines a nascent Delta function by virtue of the limit $1 /(2 \mathrm{Cn}) \operatorname{sech}^{2}(x / \mathrm{Cn}) \rightarrow \delta(x)$, a tempting replacement for $(\nabla \phi)^{2}$ in $(2.10)$ is $2 /\left(3 \mathrm{Cn}^{2}\right) \cdot\left(1-\phi^{2}\right)$ after appropriate scaling. However, as we shall see, this choice has a different behavior near the origin and it shall later be convenient to use the slightly altered scaling $1 / \mathrm{Cn}^{2} \cdot\left(1-\phi^{2}\right)$ (see Section 4.2). We thus define our "Model 2" by replacing (2.10) with

$$
F_{1}=-\frac{1}{4} \psi\left(1-\phi^{2}\right)
$$

Taking variational derivatives we obtain the new chemical potentials

$$
\begin{aligned}
& \mu_{\phi}=-\phi+\phi^{3}-\frac{\mathrm{Cn}^{2}}{2} \Delta \phi+\frac{1}{2} \psi \phi+\frac{1}{2 \mathrm{Ex}} \psi \phi, \\
& \mu_{\psi}=\operatorname{Pi} \log \frac{\psi}{1-\psi}+\frac{1}{4} \phi^{2}+\frac{1}{4 \mathrm{Ex}} \phi^{2},
\end{aligned}
$$

where it is clear that Model 2 can be implemented by simply skipping the term $F_{1}$ altogether and substitute $\mathrm{Ex} \rightarrow 1 /(1 / \mathrm{Ex}+1)$ in (2.11).

Although the square hyperbolic secant is a well-known nascent Delta function (it is the derivative of the Fermi-Dirac function), in the present context one can actually continue to use the quartic hyperbolic secant, without introducing any derivatives. A simple replacement for the square gradient achieving just this is $1 / \mathrm{Cn}^{2} \cdot\left(1-\phi^{2}\right)^{2}$. Accordingly, our "Model 3" uses, in place of (2.10),

$$
F_{1}=-\frac{1}{4} \psi\left(1-\phi^{2}\right)^{2}
$$

with the associated chemical potentials

$$
\begin{aligned}
& \mu_{\phi}=-\phi+\phi^{3}-\frac{\mathrm{Cn}^{2}}{2} \Delta \phi+\left(1-\phi^{2}\right) \psi \phi+\frac{1}{2 \mathrm{Ex}} \psi \phi, \\
& \mu_{\psi}=\operatorname{Pi} \log \frac{\psi}{1-\psi}-\frac{\left(1-\phi^{2}\right)^{2}}{4}+\frac{1}{4 \mathrm{Ex}} \phi^{2} .
\end{aligned}
$$

Evidently, Model 3 is stiffer than Model 2 and it will also be shown to produce a sharper equilibrium profile. Figure 3.1 and 3.2 below show representative sample 
simulations of all four models thus far considered. These results were obtained with the numerical method discussed in Appendix B.

3.3. Sample simulation: ill-posedness. In Figure 3.1 we compare an unstable and a stable case of the original surfactant phase-field model (referred to as "Model 0 " from now on). The initial data, in the form of a uniform surfactant profile, was chosen in accordance with the stability criterion (3.5) and in such a way that both cases are in close proximity to the boundary of the region of well-posedness. During the simulation time displayed here it holds for the numerical solution $\psi$ that $0<\psi<1$ and that the associated Ginzburg-Landau (2.6) energy is decreasing. Shortly after the displayed simulation time the numerical solution becomes negative such that the free energy formally becomes multivalued.

In Section 4.2 we more carefully evaluate the sharpness of the condition (3.5) after developing some more concepts (see Figure 4.1).

Examples of all alternative models proposed in Section 3.2 are displayed in Figure 3.2 were we simulate the ill-posed case from Figure 3.1 anew. Unlike Model 0, for this choice of parameters, all three new models are perfectly stable.

\section{ANALYSis AND NUMERICAL EXPERIMENTS IN 1D}

In order to evaluate and compare the proposed models we now proceed to derive some analytical estimates, most of which we test through numerical simulations. We discuss equilibrium solutions, adsorption isotherm relations, and we also evaluate experimentally the diffusion-controlled adsorption dynamics at the interface.

4.1. Planar equilibrium solution at constant surfactant concentration. If $\psi$ is held constant at a bulk value $\psi_{b}$, steady-state of $\phi$ implies for both our starting model (2.12) and for Model 1 in Section 3.2.1 that

$$
\mu_{\phi}=-\phi+\phi^{3}-\frac{\mathrm{Cn}^{2}}{2} \Delta \phi+\frac{\mathrm{Cn}^{2}}{2} \psi_{b} \Delta \phi+\frac{1}{2 \mathrm{Ex}} \psi_{b} \phi=0 .
$$

Generally, steady-state requires $\mu_{\phi}=$ constant, but it is not difficult to see that a solution which is odd about the origin (say, $\phi( \pm \infty)= \pm(1-\varepsilon)$ ) must in fact have $\mu_{\phi}=0$. Rewriting we get

$$
\begin{aligned}
\mu_{\phi} & =-\underbrace{\left(1-\frac{1}{2 \mathrm{Ex}} \psi_{b}\right)}_{=: \phi_{b}^{2}} \phi+\phi^{3}-\left(1-\psi_{b}\right) \frac{\mathrm{Cn}^{2}}{2} \Delta \phi \\
& =\phi_{b}^{3}[-\phi_{b}^{-1} \phi+\left(\phi_{b}^{-1} \phi\right)^{3}-\underbrace{\frac{1-\psi_{b}}{\phi_{b}^{2}} \frac{\mathrm{Cn}^{2}}{2}}_{=: \mathrm{Cn}_{\varphi}^{2} / 2} \Delta \phi_{b}^{-1} \phi] .
\end{aligned}
$$

This is just a new Cahn-Hilliard steady-state equation in the scaled variable $\varphi:=$ $\phi_{b}^{-1} \phi$ with Cahn-number $\operatorname{Cn}_{\varphi}=\operatorname{Cn} \sqrt{1-\psi_{b}} / \phi_{b}$. From the classical solution $\varphi=$ $\tanh \left(x / \mathrm{Cn}_{\varphi}\right)$ we therefore get

$$
\phi(x)=\phi_{b} \tanh \left(\phi_{b} \frac{x}{\operatorname{Cn} \sqrt{1-\psi_{b}}}\right),
$$



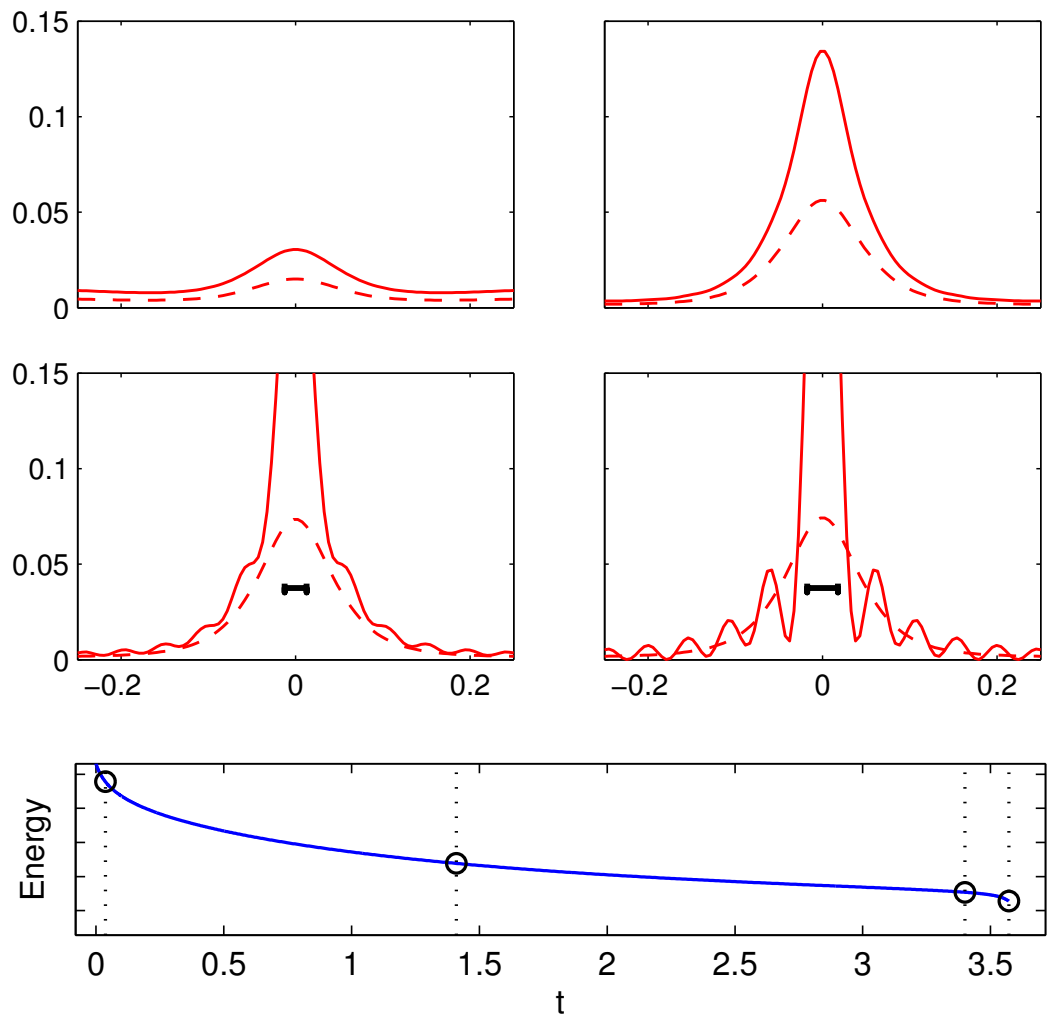

Figure 3.1. Time snapshots of the surfactant concentration for an unstable (solid) and a stable (dashed) case. All parameters are the same in the two cases $(\mathrm{Cn}=1 / 6, \mathrm{Ex}=1$, and $\mathrm{Pi}=$ 0.1227 ), but the amount of surfactant differs by a factor of two. The initial data for $\psi(x)$ is a flat profile with height 0.012 and 0.006 , respectively, and $\phi(x)$ is initially and in both cases set to $\tanh (x / \mathrm{Cn})$. In the top two graphs the instability has not yet developed. In the two graphs below the $\mathcal{O}\left(\omega^{2}\right)$-part of (3.3) is positive inside the indicated small region near the origin, and the instability immediately becomes manifest. In the bottom graph the free energy (2.6)-(2.11) is plotted as a function of time $t$ and the times for the four snapshots are indicated by circles. See text for further comments.

with

$$
\phi_{b}^{2}=1-\frac{1}{2 \mathrm{Ex}} \psi_{b}
$$

At the interface we have for this solution that $\operatorname{Cn} d / d x \phi(0)=1-(1 / \mathrm{Ex}-1) \psi_{b} / 2+$ $\mathcal{O}\left(\psi_{b}^{2}\right)$ implying that the sharpness of the interface is independent of the surfactant loading up to $\mathcal{O}\left(\psi_{b}^{2}\right)$ when $\mathrm{Ex}=1$. The bulk behavior as $x \rightarrow \pm \infty$ is $\pm \phi_{b}$ which means that the coefficient Ex controls the bulk solubility of the two phases relative to each other. Namely, with $\phi_{b}=1-\psi_{b} /(4 \mathrm{Ex})+\mathcal{O}\left(\psi_{b}^{2}\right)$ (from (4.4)), we see that 

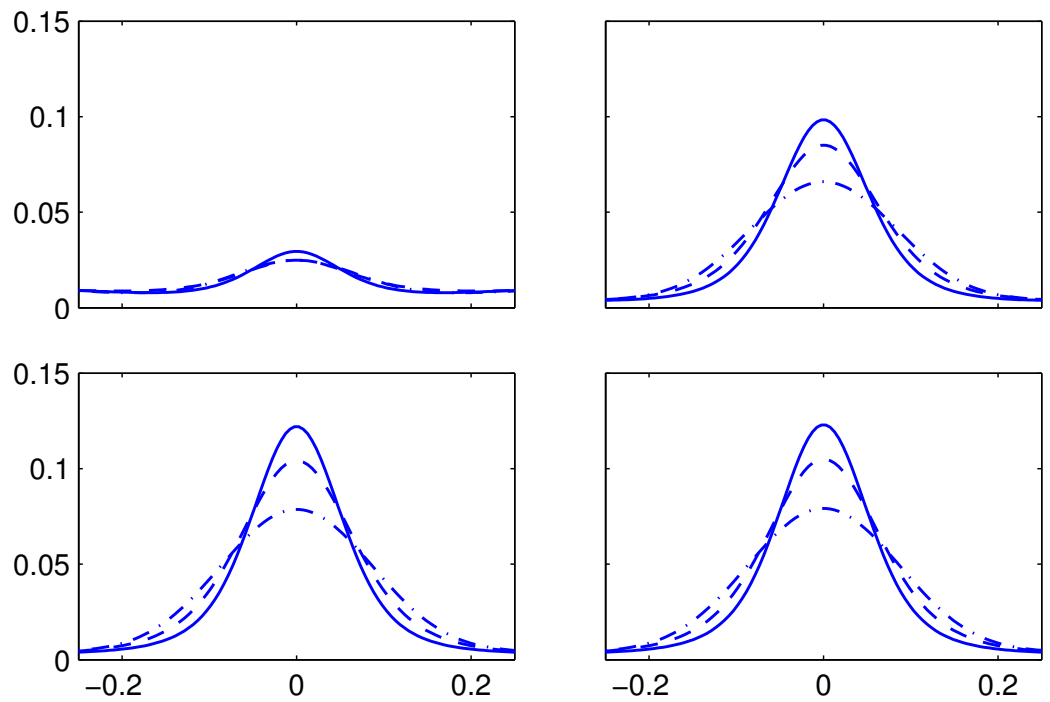

FiguRE 3.2. Snapshots and parameters as in the unstable case of Figure 3.1, but using Model 1-3 instead. Dash-dot: Model 1 with $\sigma=8 \mathrm{Pi}$, dashed: Model 2, solid: Model 3.

(3.9) becomes

$$
\left[\psi_{b}, \psi_{b} /(8 \mathrm{Ex}), 1-\psi_{b} /(8 \mathrm{Ex})\right]
$$

to within $\mathcal{O}\left(\psi_{b}^{2}\right)$ in the two phases. Hence for a system described by these thermodynamic potentials, the presence of the surfactant in the bulk will allow for a small but finite solubility of the one fluid in the other.

Proceeding in a similar fashion with Model 2 using (3.12) we obtain the equilibrium solution

$$
\begin{aligned}
\phi(x) & =\phi_{b} \tanh \left(\phi_{b} \frac{x}{\mathrm{Cn}}\right), \\
\phi_{b}^{2} & =1-\left(1+\frac{1}{\mathrm{Ex}}\right) \frac{\psi_{b}}{2},
\end{aligned}
$$

and where $\operatorname{Cn} d / d x \phi(0)=\phi_{b}^{2}=1-\mathcal{O}\left(\psi_{b}\right)$ and is $<1$ for any positive value of Ex.

Finally, for Model 3 using (3.15) we get after similar manipulations

$$
\begin{aligned}
\phi(x) & =\phi_{b} \tanh \left(\phi_{b} \frac{x}{\operatorname{Cn} \sqrt{1-\psi_{b}}}\right), \\
\phi_{b}^{2} & =\frac{1-\left(1+\frac{1}{2 \mathrm{Ex}}\right) \psi_{b}}{1-\psi_{b}} .
\end{aligned}
$$

Not only are (4.8) and (4.3) identical in form, but (4.9) and (4.4) in fact also agree up to order $\mathcal{O}\left(\psi_{b}^{2}\right)$. Consequently we have again that $\operatorname{Cn} d / d x \phi(0)=1-$ $(1 / \mathrm{Ex}-1) \psi_{b} / 2+\mathcal{O}\left(\psi_{b}^{2}\right)$ so that for $\mathrm{Ex}=1$, the sharpness of the interface is independent of the surfactant loading up to $\mathcal{O}\left(\psi_{b}^{2}\right)$.

Conclusion. For all four models, the parameter Ex controls the bulk solubility. Model 0, 1, and 3 all agree closely in terms of the bulk value $\phi_{b}$ and also in the 
sharpness of the phase-field interface $\phi=0$. By comparison, Model 2 has a more diffuse interface.

4.2. Adsorption isotherm. We now turn our attention to equilibrium profiles for $\psi$, following the line of reasoning in [44] closely. Since at steady-state one must have that the chemical potential is constant, the basic approach is to solve the equation $\mu_{\psi(x)}=\mu_{\psi_{b}}$ for $\psi$, where $\psi_{b}$ as before is the bulk concentration $\psi(\infty)$. We write the chemical potentials for all four models in the forms

$$
\begin{aligned}
\mu_{\psi_{b}} & =\operatorname{Pi} \log \frac{\psi_{b}}{1-\psi_{b}}+B_{b}+\frac{1}{4 \mathrm{Ex}} \phi_{b}^{2}, \\
\mu_{\psi(x)} & =\operatorname{Pi} \log \frac{\psi}{1-\psi}+B+\frac{1}{4 \mathrm{Ex}} \phi^{2} .
\end{aligned}
$$

Subtracting and introducing the intermediate variable $\psi_{c}(x)$ we get the relation

$$
\text { Pi } \log \psi_{c}(x)=B-B_{b}-\frac{1}{4 \operatorname{Ex}}\left(\phi_{b}^{2}-\phi^{2}\right)
$$

in terms of which the steady-state profile is given by

$$
\psi(x)=\frac{\psi_{b}}{\psi_{b}+\psi_{c}(x)\left(1-\psi_{b}\right)}=\frac{\psi_{b}}{\psi_{b}+\psi_{c}(x)}+\mathcal{O}\left(\psi_{b}\right) .
$$

The difference $B-B_{b}$ is given by, respectively,

$$
B-B_{b}=\left\{\begin{array}{ll}
-\frac{\mathrm{Cn}^{2}}{4}(\nabla \phi)^{2} & (\text { Model 0) } \\
-\frac{\mathrm{Cn}^{2}}{4}(\nabla \phi)^{2}-\frac{\mathrm{Cn}^{2}}{2} \Delta \psi-\frac{\sigma}{2}\left(\psi-\psi_{b}\right) & (\text { Model 1) } \\
-\frac{1}{4}\left(\phi_{b}^{2}-\phi^{2}\right) & (\text { Model 2) } \\
-\frac{1}{4}\left[\left(\phi_{b}^{2}-\phi^{2}\right)\left(2-\phi_{b}^{2}-\phi^{2}\right)\right] & (\text { Model 3) }
\end{array} .\right.
$$

Clearly, for Model 1, this line of reasoning needs to be augmented with additional assumptions or estimates in order not to be circular. For the other cases, with a fixed phase-field profile $\phi(x) \approx \phi_{b} \tanh (x / \mathrm{Cn}$ ) (as determined in Section 4.1), (4.13) yields a quite decent approximation as we shall see.

Specializing $x$ in (4.13) to the origin we get

$$
\begin{aligned}
\psi_{0} & =\frac{\psi_{b}}{\psi_{b}+\psi_{c}}+\mathcal{O}\left(\psi_{b}\right), \\
\text { Pi } \log \psi_{c} & =-\frac{1}{4}\left(1+\frac{1}{\mathrm{Ex}}\right)+\mathcal{O}\left(\psi_{b}\right) .
\end{aligned}
$$

To arrive at (4.16), for Model 0 we have to assume that $\mathrm{Cn}^{2}(\nabla \phi(0))^{2}=1+\mathcal{O}\left(\psi_{b}\right)$ (cf. Section 4.1), while for Model 2 and 3 we only need to use the fact that $\phi(0)=0$.

Eq. (4.15) is the Langmuir isotherm and $\psi_{c}$ as defined by (4.16) is the Langmuir (equilibrium) adsorption constant. The fact that all models except Model 1 possesses the same Langmuir isotherm makes them comparable and also explains our special choice of scaling when constructing Model 2 in Section 3.2.2.

For completeness, we note that (4.13) for Model 1 becomes as $x \rightarrow 0$,

$$
\psi_{0}=\frac{\psi_{b}}{\psi_{b}+\psi_{c} \exp \left(-\alpha \psi_{0}\right) R}+\mathcal{O}\left(\psi_{b}\right),
$$

with $\psi_{c}$ still defined by (4.16), and where $\alpha=\sigma /(2 \mathrm{Pi}), R=\exp \left(-\mathrm{Cn}^{2} \Delta \psi_{0} /(2 \mathrm{Pi})\right)$. With $R=1,(4.17)$ is the Frumkin isotherm which essentially is an effect of the presence of the lateral interaction term $-\sigma / 4 \cdot \psi^{2}$ in (3.6). Unfortunately, there 


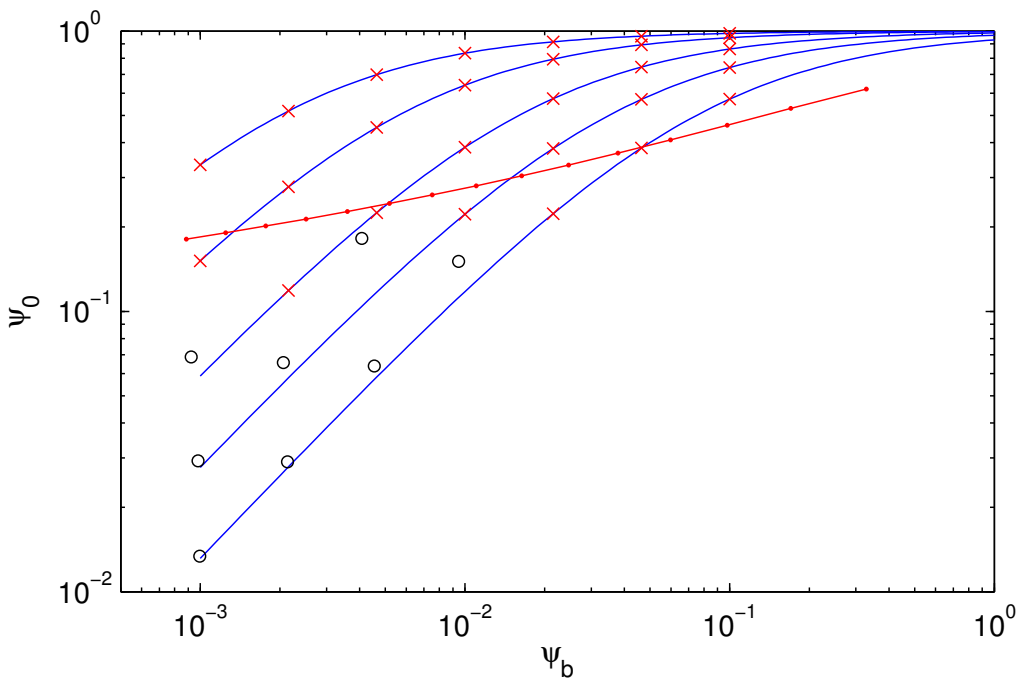

Figure 4.1. Attempt to verify the Langmuir isotherm (4.15) through numerical simulations of Model 0 . This figure is intended to be an exact reproduction of the results in [44, Fig. 2]. However, here we clearly see that the model is ill-posed as predicted by the theory in Section 3.1. Solid: the isotherm (4.15) for different values of $\psi_{c}$, circles: numerical values. The dotted line is the sufficient condition for ill-posedness (3.5) and crosses are used to denote (missing) unphysical solutions (e.g. large negative values).

is no evident relation between $\psi_{0}$ and $\Delta \psi_{0}$ which can be used to close this line of reasoning. Therefore, for Model 1 the adsorption isotherm cannot be explicitly determined.

4.2.1. Numerical isotherm. Using our one-dimensional spectral Galerkin code as outlined in Appendix B we have performed several numerical experiments with the adsorption isotherm relation. For all experiments in this section we used the same set of parameters: $\mathrm{Cn}=1 / 6, \mathrm{Ex}=1, \psi_{c} \in\{0.0020,0.0056,0.016,0.035,0.075\}$ (with Pi determined from the relation (4.16)), and with $\psi_{b}$ sampled in the interval $\left[10^{-3}, 10^{-1}\right]$. All parameters have been chosen to agree with those in [44, Fig. 2]. The simulations were started with the profile $\phi(x)=\tanh (x / \mathrm{Cn})$ with $\psi(x)$ defined by (4.13) and, for convenience, $\phi_{b} \equiv 1$. Finally, for Model 1 we used the value $\sigma=8 \mathrm{Pi}$, obtained from the requirement that the non-gradient part of $F_{\psi}$ be convex (see (3.10)) and otherwise simply by trial and error to approximately match the visual appearances of the profiles for Model 2 and 3 (cf. Figure 4.4).

In Figure 4.1 we numerically test the sharpness of our analysis of Model 0 in Section 3.1. Clearly, the model is ill-posed for most of the parameters tested here, rendering the model very questionable. From the figure, it is also seen that the sufficient condition for ill-posedness (3.5) is quite sharp.

In Figure 4.1 a numerical isotherm is obtained for Model 1. The Langmuir isotherm is not a bad model for small values of $\psi_{c}$, but the measured adsorption breaks off for larger values and also for higher surfactant concentrations. A few cases 


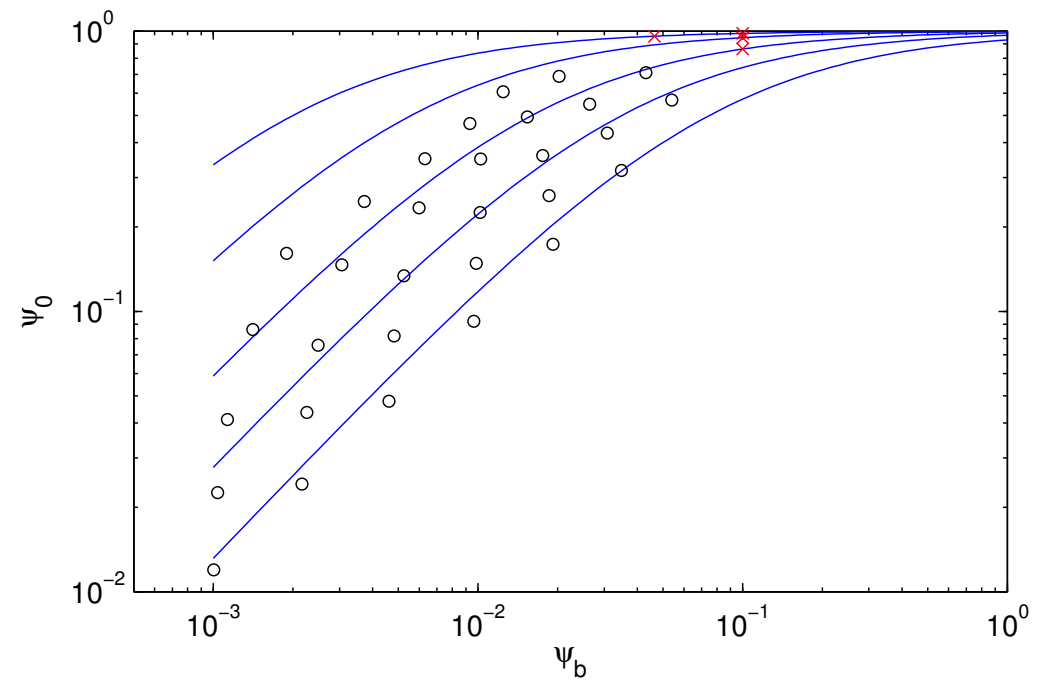

FiguRE 4.2. Results from simulations with parameters as in Figure 4.1 but using Model 1 instead. Since there is no known adsorption relation in this case, the Langmuir isotherm (4.15) in solid is displayed for reference only. A total of four unstable cases were detected (crosses).

were found to be numerically unstable by producing an extremely sharp profile for $\phi$. We have not been able to analyze this and do not know at present if this is an actual property of the model or a numerical artifact of some kind.

In Figure 4.3 we similarly compare the theoretical isotherm (4.15) with numerical values for Model 2 and 3. No unstable cases were detected and the results are all in very good agreement. For Model 2, the initial data used in the experiments were a bit off the actual equilibrium causing the measured values to "creep" slightly. However, all values stay in close proximity to the predicted isotherm curve.

Finally, in Figure 4.4 the initial approximate surfactant concentration profile is compared to the final equilibrium profile for a single value of $\psi_{c}$ and for different surfactant bulk concentrations $\psi_{b}$.

Conclusion. We verified the theoretical analysis in Section 3.1 and in particular the sharpness of the sufficient condition for ill-posedness (3.5). The Langmuir isotherm (4.15) for Model 0 as published originally in [44] and again, using altered parameters, in [36] could therefore not be obtained. Model 1, although stable in most cases, does not a priori possess a natural adsorption isotherm. Model 2 and 3 are not only stable, but they also satisfy the Langmuir isotherm very accurately. Model 3 produces the most sharp surfactant profile and Model 1 yields the most damped profile, at least towards the higher values of surfactant concentrations tested here. The analytical prediction (4.13) is very accurate for Model 2 and 3.

4.3. Adsorption dynamics. The adsorption dynamics for an interface in contact with a semi-infinite bulk was considered in the early paper [53] by Ward and Tordai. Here the time-dependent decrease of surface tension under the presence of a solute was explained on the basis of diffusion. Their approach has later been refined; 

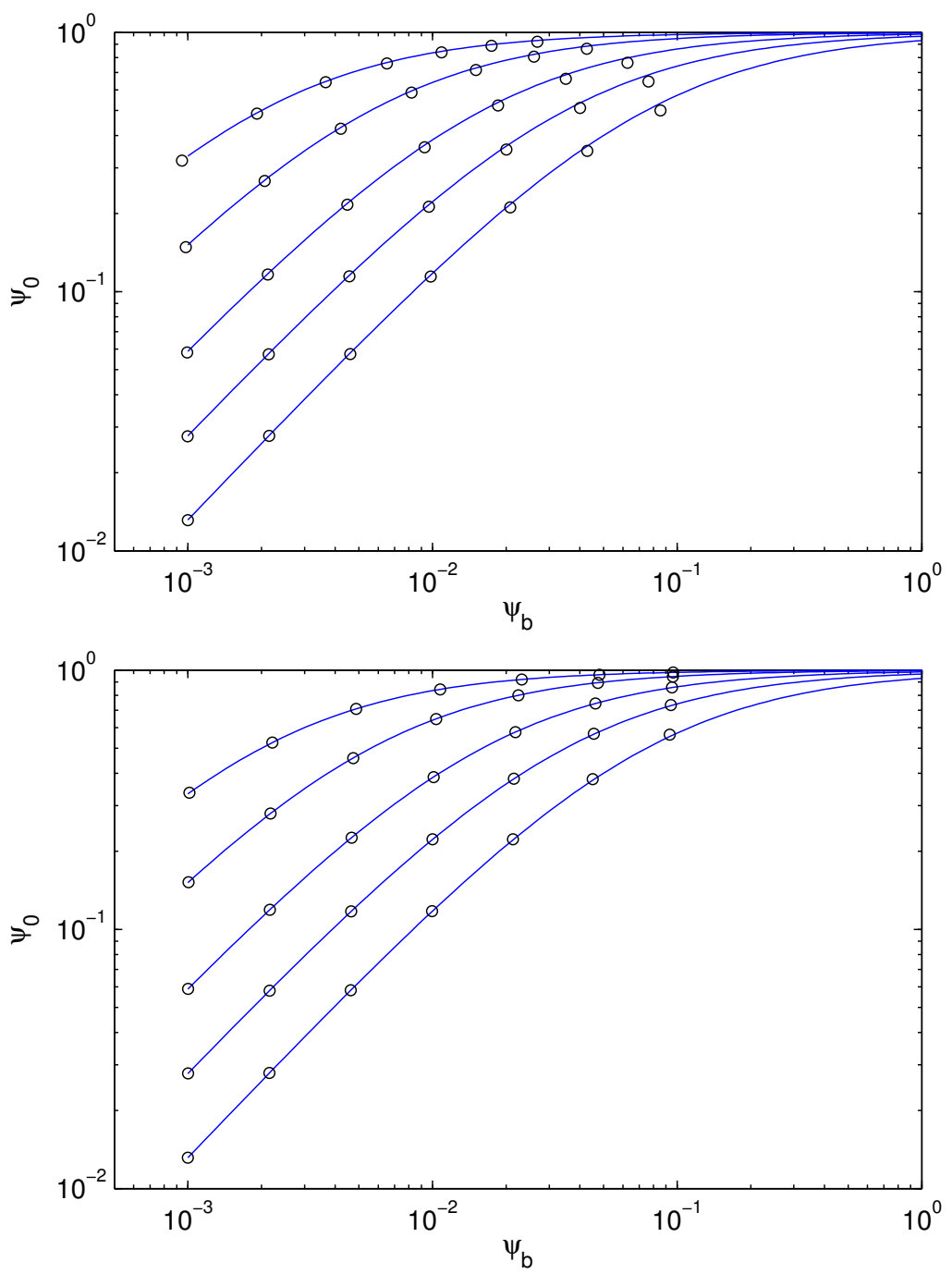

FiguRE 4.3. Langmuir adsorption isotherm for Model 2 (top) and 3 (bottom).

notably by Hansen [23] who included also the process of evaporation of solute from the interface, and by Ariel, Diamant, and Andelman [9, 10] who proposed a separate treatment for ionic surfactants.

In order to cohere with this classical set-up we continue to use our one-dimensional setting with an interface at $x=0$. We treat the phase-field variable $\phi$ as being time-independent in the arguments below; the precise form is not critical as long as the scaling is such that the interface is located within $|x| \lesssim \mathrm{Cn}$ and such that $|\phi(x)| \sim 1$ for $|x| \gg$ Cn. As before we assume the constant scalar bulk concentration $\psi_{b}=\psi(t, x \rightarrow \infty)$ and further put $\psi_{0}(t):=\psi(t, x=0)$, and also $\psi_{0, \mathrm{eq}}:=\psi_{0}(t \rightarrow \infty)$. 


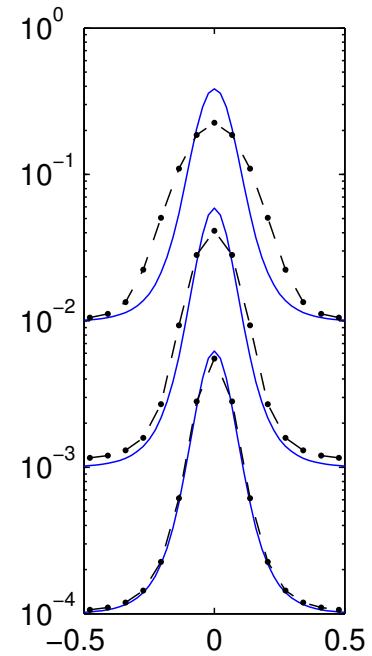

(a) Model 1.

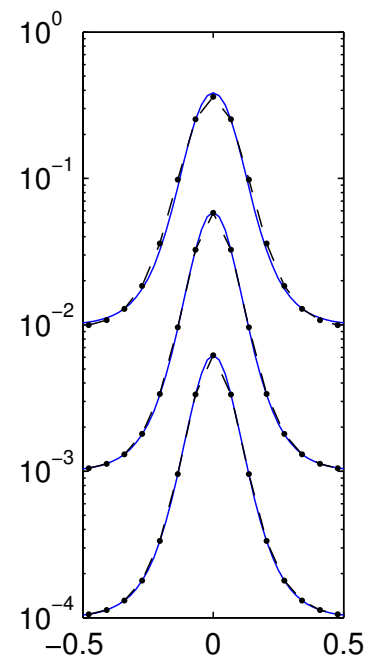

(b) Model 2.

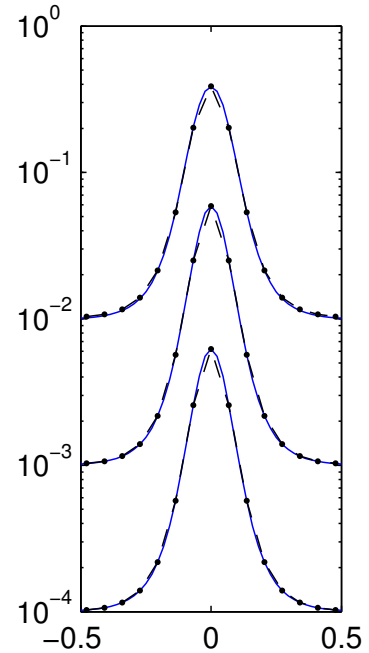

(c) Model 3.

FiguRE 4.4. Initial surfactant concentration profiles according to (4.13) (dashed) and numerical equilibrium values (solid) for different values $\psi_{b} \in\left\{10^{-4}, 10^{-3}, 10^{-2}\right\}$ and $\psi_{c}=0.016$. For Model 1 the value of $B-B_{b}$ in (4.14) formally belonging to Model 0 was used in order to obtain a closed expression for the initial profile.

The model proposed in [10] is "sharp" in the sense that it is directly formulated as a discrete model with a characteristic length-scale $a$ (compartment size) on the order of a single molecule. We may therefore refer to such a model as "microscopic" and it is clear that any continuous model will have difficulties as the sharpness of the interface approaches this length-scale. Indeed, for the diffuse interface model under current consideration, the strict limit $\mathrm{Cn} \rightarrow 0$ does not make sense as the limiting equilibrium surfactant concentration profile necessarily becomes a constant bulk-value with a single point removed at the interface itself (this follows from the isotherm relation (4.13)). However, the limiting behavior as $\mathrm{Cn} \rightarrow a \ll 1$ is still of interest since it makes a dynamic comparison with the microscopic model in [10] possible. Specifically, we are interested in testing the adsorption dynamics at the interface for which there are known discrete modeling approximations available.

In order to estimate the behavior as $\mathrm{Cn} \rightarrow a$, where we may think of $a>0$ as the length-scale of a single molecule, we write the surfactant concentration in terms of inner and outer variables,

$$
\begin{aligned}
& \psi(t, x)=\psi_{\text {in }}(t, y) \quad \text { for }-1 \leq y \leq 1, \text { with } y:=x / \mathrm{Cn}, \\
& \psi(t, x)=\psi_{\text {out }}(t, x) \quad \text { for }|x|>\mathrm{Cn} .
\end{aligned}
$$

Using the constant phase-field profile $\phi(x)=\tanh (x / \mathrm{Cn})$ we can write the PDE (2.2) for $\psi$ as

$$
\frac{\partial \psi}{\partial t}=\frac{\mathrm{Pi}}{\mathrm{Pe}_{\psi}} \frac{\partial^{2} \psi}{\partial x^{2}}+\frac{1}{\mathrm{Pe}_{\psi}} \frac{\partial}{\partial x}\left[\psi(1-\psi) \varphi^{\prime}(x)\right]
$$


in terms of which

$$
\varphi(x)=\left\{\begin{array}{lr}
-\frac{\left(1-\phi^{2}\right)^{2}}{4}+\frac{\phi^{2}}{4 \mathrm{Ex}} & \text { (Model 0 and 3) } \\
-\frac{\left(1-\phi^{2}\right)^{2}}{4}-\frac{\mathrm{Cn}^{2}}{2} \psi^{\prime \prime}-\frac{\sigma}{2} \psi+\frac{\phi^{2}}{4 \mathrm{Ex}} & (\text { Model 1) } \\
\frac{\phi^{2}}{4}+\frac{\phi^{2}}{4 \mathrm{Ex}} & (\text { Model 2) }
\end{array} .\right.
$$

For $|x| \gg \mathrm{Cn}$ and in all four cases we readily get the bulk diffusion equation,

$$
\frac{\partial \psi_{\text {out }}}{\partial t}=\frac{\mathrm{Pi}}{\mathrm{Pe}_{\psi}} \frac{\partial^{2} \psi_{\text {out }}}{\partial x^{2}}
$$

Also, through the change of variables $y=x / \mathrm{Cn}$ expressed in (4.18), we get the inner dynamics

$$
\frac{\partial \psi_{\text {in }}}{\partial t}=\frac{\mathrm{Pi}}{\mathrm{Cn}^{2} \mathrm{Pe}_{\psi}} \frac{\partial^{2} \psi_{\text {in }}}{\partial y^{2}}+\frac{1}{\mathrm{Cn}^{2} \mathrm{Pe}_{\psi}} \frac{\partial}{\partial y}\left[\psi_{\text {in }}\left(1-\psi_{\text {in }}\right) \varphi^{\prime}(y)\right]
$$

where for Model 1 the change of variables implies

$$
\varphi(y)=-\frac{\left(1-\phi^{2}\right)^{2}}{4}-\frac{\psi^{\prime \prime}}{2}-\frac{\sigma}{2} \psi+\frac{\phi^{2}}{4 \mathrm{Ex}} .
$$

Assuming an asymptotic expansion of the form $\psi_{\text {in }}(t, y) \sim \sum_{j>0} \mathrm{Cn}^{j} \psi_{j, \mathrm{Cn}}(t, y)$ we have to leading order in $\mathrm{Cn}$ that the inner variable is in equilibrium with the outer variable via the Dirichlet (matching) boundary condition $\psi_{\text {in }}(t, \pm 1)=\psi_{1}(t):=$ $\psi_{\text {out }}(t, \pm \mathrm{Cn})$. As in $[9,10]$ we may interpret the new variable $\psi_{1}$ as the concentration in the sub-surface layer and we have explicitly enforced an even symmetry $\psi(t, x)=\psi(t,-x)$ in order to remain compatible with the modeling in those references.

We connect the two variables by requiring that the total volume is conserved (assuming conservative outer boundary conditions),

$$
\frac{d V}{d t}=\int_{|x| \geq \mathrm{Cn}} \frac{\partial \psi_{\text {out }}}{\partial t} d x+\int_{-1}^{1} \frac{\partial \psi_{\text {in }}}{\partial t} \operatorname{Cn} d y=-\frac{2 \mathrm{Pi}}{\mathrm{Pe}_{\psi}} \psi_{1}^{\prime}+\frac{d}{d t} \int_{-1}^{1} \psi_{\text {in }} \operatorname{Cn} d y=0 .
$$

For Model 2 and 3, with $\psi_{\text {in }}$ in equilibrium the inner variable can in principle be determined explicitly as a function of $\psi_{1}$ thanks to an isotherm-like relation. Model 1 does not possess such an isotherm relation, but in this case a steady-state solution of the inner dynamics (4.23) plays the same role. Although an analytic expression for the resulting integral is lacking we argue that under the present scaling, the near surface dynamics is essentially of discrete character in the two variables $\psi_{0}$ and $\psi_{1}$. As an example, using the trapezoidal rule we get the approximative relation

$$
\frac{\partial \psi_{0}}{\partial t}+\frac{\partial \psi_{1}}{\partial t}=\frac{2 \mathrm{Pi}}{\mathrm{Pe}_{\psi} \mathrm{Cn}} \psi_{1}^{\prime} .
$$

Interestingly, (4.22) and (4.26) above correspond directly to Eqs. (2.11) and (2.12) in the derivation in [9]. These equations can be solved analytically in terms of an integral relation such that asymptotic estimates can be obtained. More precisely, when starting with a uniform profile $\psi(t=0, x)=\psi_{b}$ we have the asymptotic 
"footprint" of diffusion [10],

$$
\frac{\psi_{0}(t)}{\psi_{0, \mathrm{eq}}} \sim 1-\left(\frac{\tau_{0}}{t}\right)^{1 / 2}, \quad t \rightarrow \infty .
$$

There is also an initial transient phase with a $\sqrt{t}$-dependence [23],

$$
\psi_{0}(t) \sim \text { const. }+\left(\frac{t}{\tau_{1}}\right)^{1 / 2} .
$$

Finally, as pointed out in [10], with a uniform initial profile there is also an ultrashort linear transient before (4.28) is valid,

$$
\frac{\psi_{0}(t)}{\psi_{b}} \sim 1+\text { const. } \times t .
$$

4.3.1. Numerical dynamics. This quite interesting typical adsorption behavior is displayed in Figure 4.5, again using the numerical method outlined in Appendix B. The initial very short linear transient is the phase where the region just outside the interface quickly becomes depleted of surfactant. In the following $\sqrt{t}$-dependent phase, surfactant diffuses from outside the sub-surface region, enters it, and then immediately adsorbs to the interface, keeping the concentration in the sub-surface region in equilibrium. The asymptotic regime occurs towards the end of this process when the interface gradually becomes saturated and the adsorption therefore slows down.

In Figure 4.6 and 4.7 we numerically fit (using standard polynomial least squares) the measured time-dependent interfacial adsorption to the theoretical expressions for a sharp interface expressed in (4.27)-(4.29). Although the two models display some differences, both versions clearly fit quite well with the theoretical predictions. Qualitatively similar results were produced also for Model 1, but the temporal scaling is slightly different here. Also, in one of the cases tested, we were unable to obtain a solution due to instabilities and so we choose not to report these results here.

Conclusion. The Ward-Tordai governing equations (4.22) and (4.26) are approximately retrieved by performing a multiscale analysis in terms of inner and outer variables. Experimentally, for Model 2 and 3 we verified good agreement as to the theoretical behavior in the sharp interface limit.

\section{NUMERICAL METHOD AND EXPERIMENTS IN 2D}

In this section we perform a final qualitative computational experiment with the full hydrodynamic set of equations (2.1)-(2.4). The purpose is to show that the improved model can capture the nontrivial coupling between the fluid flow and the surface forces. This is an essential property as it involves the convective redistribution of surfactants, the resulting gradients of surface tension that will appear, and a subsequent modification of the overall dynamics. One very generic example which we have chosen to study is to show that the presence of surfactant in a high enough concentration may inhibit droplet coalescence.

We present the results from simulations in two spatial dimensions: the extension to $3 \mathrm{D}$ would involve straightforward extensions of the operators in the model equations. With the obvious exception of a computationally heavier solution procedure, 


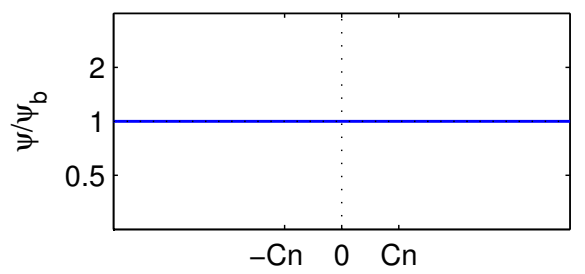

(a)

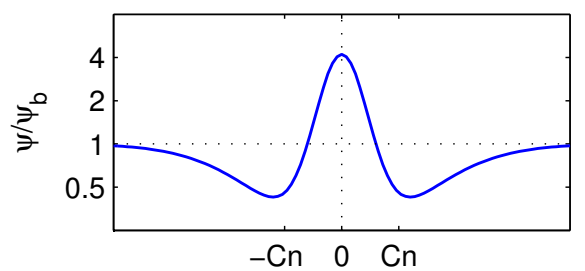

(c)

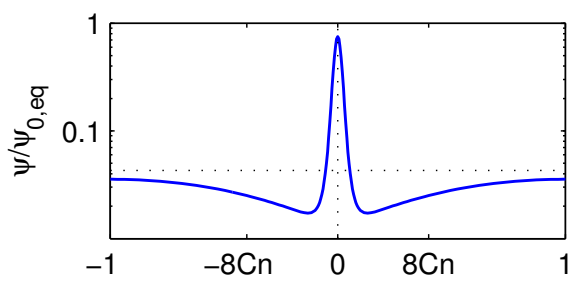

(e)

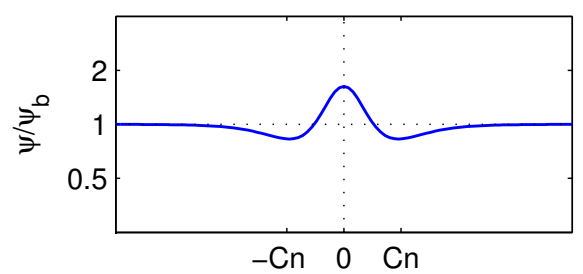

(b)

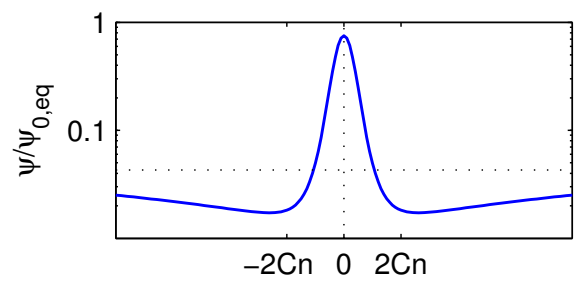

(d)

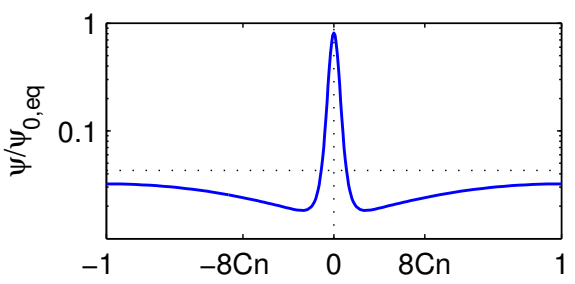

(f)

Figure 4.5. Adsorption phases. Top: starting from a uniform concentration in (a), the interface very quickly drains the subsurface region in (b)-(c). Middle: surfactant now diffuses inwards from the bulk while the concentration at the interface steadily builds up in (c)-(d). Bottom: in (e), the interface starts to reach saturation and in (f), finally, boundary effects due to the finite size of the numerical domain become prominent. In this example Model 3 was used with parameters $\mathrm{Cn}=1 / 20, \psi_{c}=0.016, \mathrm{Ex}=1$, and $\psi_{b}=10^{-2}$. Note the logarithmic vertical scale.

we do not foresee any other particular differences, since the main physical effects of surface stretching, convection of surfactant, diffusion, adsorption and desorption are present also in the $2 \mathrm{D}$ test cases we have made.

Given the previous analytical and computational results we restrict the experiment to Model 3 with chemical potentials given by (3.15)-(3.16). Although we did observe the ill-posedness for Model 0 here as well, those results are not detailed here.

5.1. Adaptive finite element method. Successful finite element methods for the Cahn-Hilliard equations have been devised by several authors. For a fully discrete convergent method targeting the fully coupled hydrodynamic flow, see [29]. 

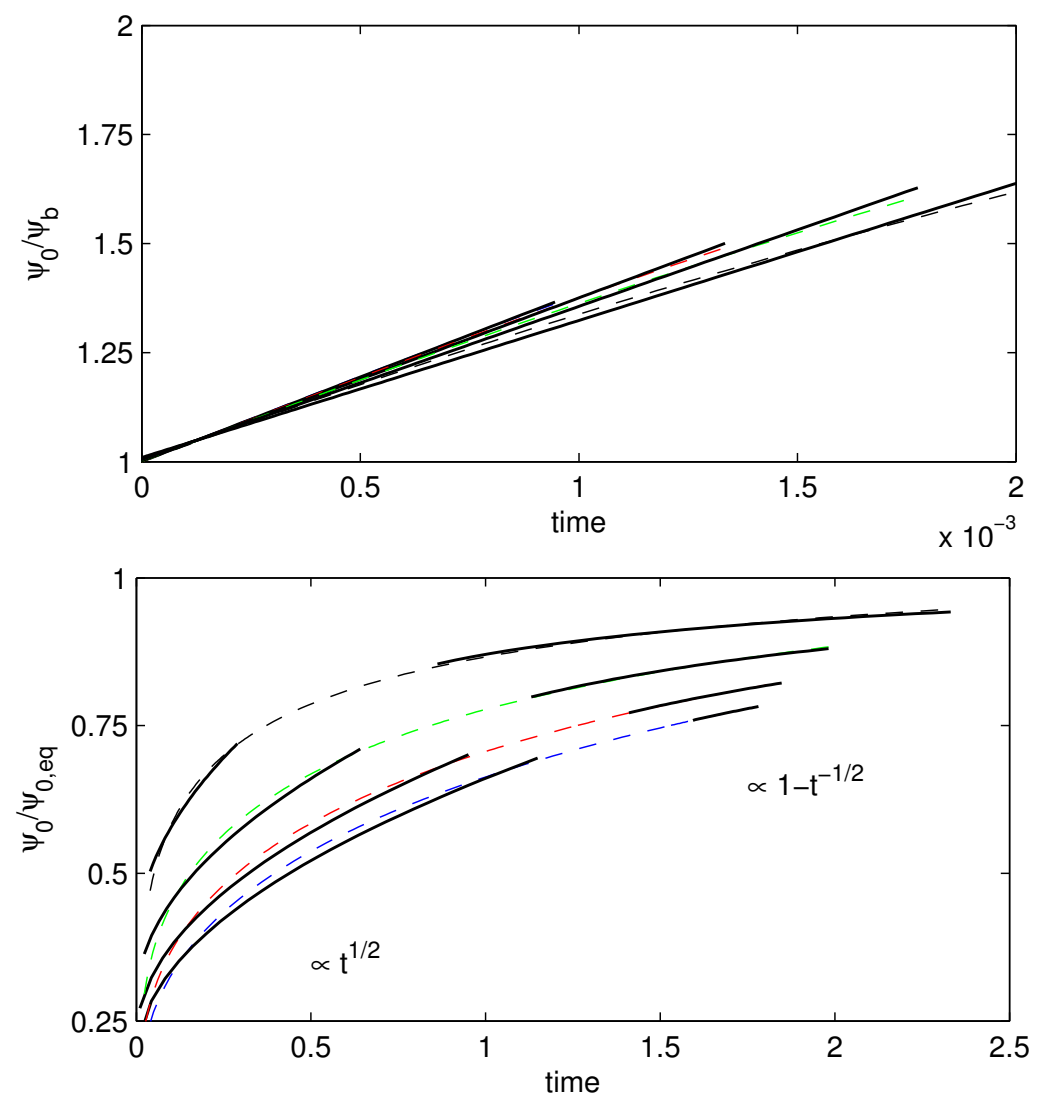

FiguRE 4.6. Top: initial very short linear transient, and bottom: the characteristic diffusion-controlled $t^{1 / 2}$-transient together with the $t^{-1 / 2}$-asymptotics. Dashed: numerical values, solid: leastsquares fit to the theoretical models (see text). These results are for Model 2 with parameters $\mathrm{Cn}=1 / 20, \psi_{c}=0.016, \mathrm{Ex}=1$. Counting from below in the bottom graph we have $\psi_{b}=[1,2,4,8] \times$ $10^{-2}$ (in the top graph the order is the reversed).

Versions with degenerate mobilities are more difficult to analyze such that $[2,6]$ are two notable exceptions. Here, although only the no-flow case is treated, on the other hand logarithmic terms are allowed in the free energy.

We carried out our numerical simulations using femLego [12], a software to solve general partial differential equations with adaptive finite element methods. The PDEs, the boundary conditions, the initial data, and the method of solving each equation are all specified in a Maple worksheet such that exact integration and differentiation are possible.

The Cahn-Hilliard-type equations (2.1)-(2.2) with chemical potentials according to (3.15)-(3.16) are treated as a coupled system for the potentials $\mu_{\phi}$ and $\mu_{\psi}$ and the composition variables $\phi$ and $\psi$. All equations are discretized in space with piecewise linear functions and in time using the trapezoidal rule, but with $\mathbf{u}$ frozen at the previous time-step, thus resembling the strategy in [29]. The coupled system 

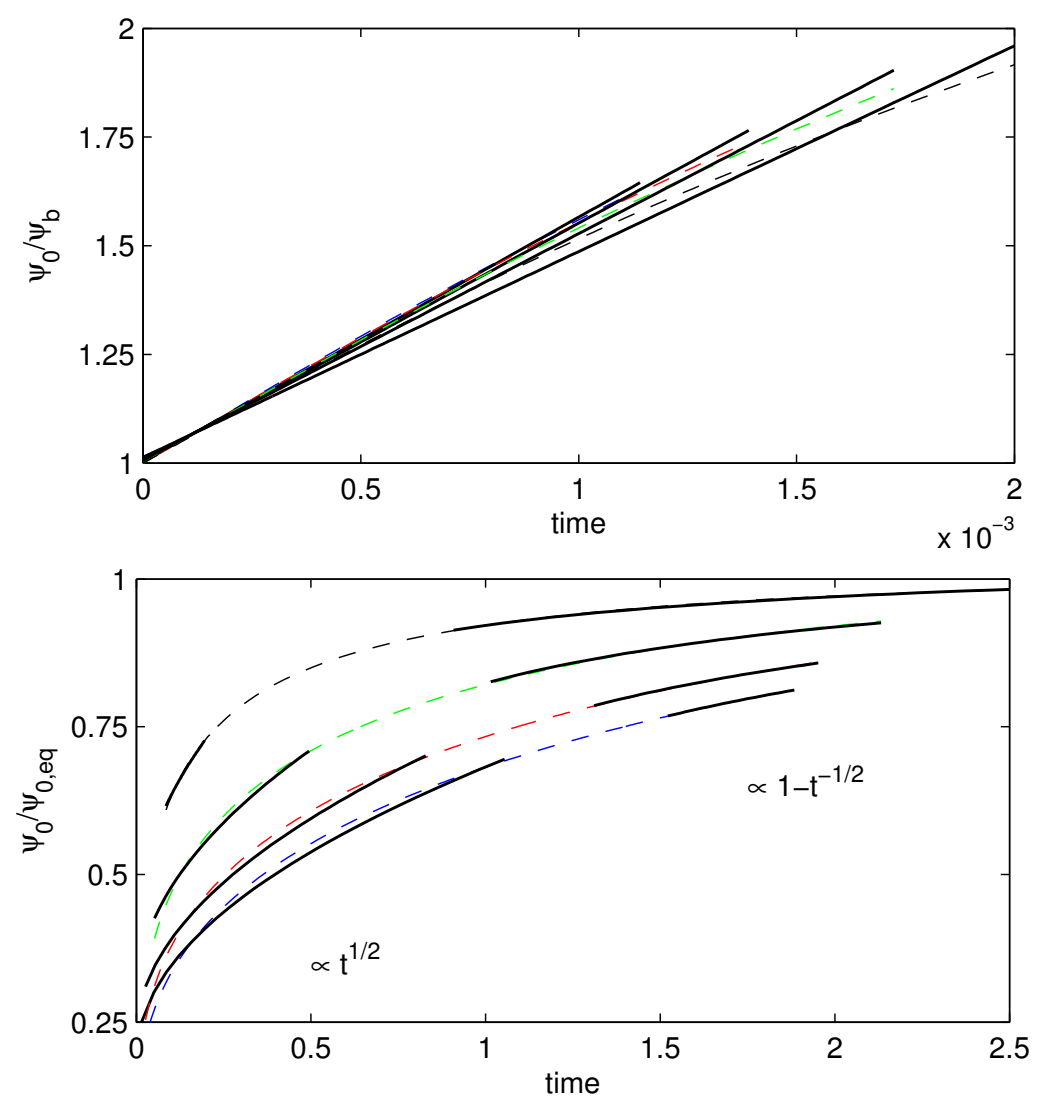

Figure 4.7. As in Figure 4.6 but for Model 3 instead. Evidently, this model enjoys a slightly faster initial adsorption at the same Langmuir-controlled final saturation.

of equations is solved using Newton iterations with UMFPACK [8] as the inner linear solver.

To ensure mesh resolution in the vicinity of the interface, an adaptively refined and derefined mesh is used with an ad hoc error criterion for each element $\Omega_{k}$,

$$
\int_{\Omega_{k}}\|\nabla \phi\|^{2} \leq \mathrm{TOL}
$$

The mesh adaptivity is implemented by marking element $\Omega_{k}$ for refinement if the element size is still larger than the minimum mesh size allowed, $h>h_{\min }$, and it does not meet the criterion (5.1). In the case that an element does meet the criterion, it is marked for derefinement unless it is an element of the initial mesh. In the experiment reported here we adjusted TOL and $h_{\min }$ so that $\geq 10$ triangles were used across the interfaces, defined here to be the regions where $\phi$ varies between -0.98 to 0.98 . Some more details about this scheme can be found in [12].

The Navier-Stokes equations (2.3)-(2.4) are solved using the projection method devised in [18]. Again, piecewise linear basis functions are used to discretize space. Firstly, an approximate pressure is extrapolated from the previous time-step and 
used when solving the momentum equation. Since this by far is the most costly step, we employed an iterative linear solver for this part [17, GMRES, Chap. 3.2]. Secondly, a projection step in the form of a Poisson equation derived from (2.3) is solved to correct the velocity.

5.2. Law of Laplace. In order to test our 2D code we first performed numerical experiments for the pure Cahn-Hilliard part (that is, $\psi=0$ in (2.1)-(2.4)). Specifically, for a single droplet in equilibrium we measured the pressure jump for different Cahn numbers and different mesh spacings. The Cahn number is the ratio between the width of the diffuse interface and the characteristic length scale in the flow, here being the droplet diameter $d$. We kept $\mathrm{Ca}=1, \mathrm{Pe}_{\phi}=3 \times 10^{-3}$, and $\mathrm{Re}=1$ fixed throughout these computations. According to Young-Laplace's equation this gives an analytical pressure difference $\Delta P=8 \sqrt{2} / 3$. The numerical domain was taken to be a cube of size $2 d \times 2 d \times 2 d$ and an equidistant mesh was used. Table 5.1 shows the relative error between the computed and the analytical pressure prediction for different Cahn numbers and mesh spacings $h$, after equilibrium has been approximately reached. Overall we observe a good agreement between the numerical and the analytical predictions. Although the error in the pressure depends on the numerical resolution of the interface we do get a fairly accurate solution even with a comparably thick interface.

\begin{tabular}{lrrrrrrr}
\hline Cn & 0.015 & 0.04 & 0.04 & 0.06 & 0.06 & 0.08 & 0.08 \\
\hline$h$ & 0.003 & 0.013 & 0.02 & 0.02 & 0.03 & 0.027 & 0.04 \\
$P_{\text {error }}$ & $0.06 \%$ & $0.6 \%$ & $2.0 \%$ & $0.6 \%$ & $1.8 \%$ & $0.9 \%$ & $1.7 \%$ \\
\hline
\end{tabular}

TABLE 5.1. Deviation between the numerical and analytical pressure for different Cahn numbers and mesh resolutions. Here $h$ is the (uniform) mesh spacing and $P_{\text {error }}$ is the relative error in the pressure jump.

5.3. Colliding droplets. To demonstrate that our extended model of surfactant adsorption can be coupled to hydrodynamics, we have used Model 3 to perform simulations of two droplets colliding in a linear shear flow channel. Figure 5.1 shows the evolution of the two droplets with droplet Reynolds number Re $=0.5$, capillary number $\mathrm{Ca}=0.1$, Péclet numbers $\mathrm{Pe}_{\phi}=10, \mathrm{Pe}_{\psi}=100$, and surfactant bulk concentration $\psi_{b} \in\left\{10^{-3}, 10^{-4}, 10^{-5}\right\}$. The parameters of the surfactant model for this simulation are given by $\mathrm{Cn}=1 / 20, \mathrm{Ex}=0.117$, and $\mathrm{Pi}=0.5857718$. All three cases are plotted on top of each other; since increasing the amount of surfactant effectively hinders coalescence of the droplets they are easily sorted out. For example, with the smallest value of bulk concentration $\psi_{b}=10^{-5}$, the two droplets have coalesced already at $t=165$. For $\psi_{b}=10^{-4}$, they coalesce at $t=190$ and they stay separate indefinitely whenever $\psi_{b} \geq 10^{-3}$. Due to a comparably strong surface tension effect and a weak fluid flow, we do not see a noticeable effect on the droplet's behavior before they are in close proximity to each other. However, when two droplets do approach, the fluid flow becomes stronger and much more complex as shown in Figure 5.2.

Figure 5.4 shows a contour plot of the surfactant concentration of two colliding droplets in the simple shear flow with $\psi_{b}=10^{-3}$. Initially, the increased pressure in 

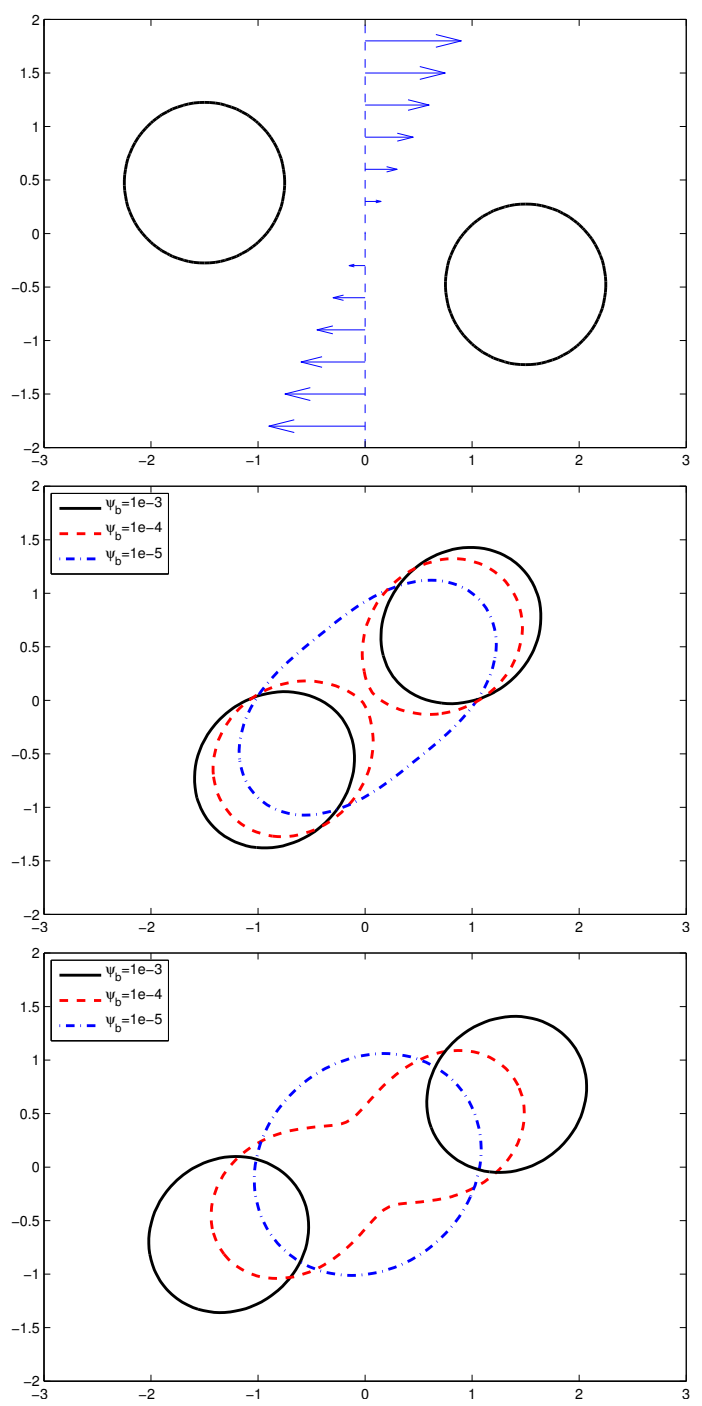

Figure 5.1. Evolution of two droplets in linear shear flow in a channel. Three cases with surfactant bulk concentration $\psi_{b} \in$ $\left\{10^{-3}, 10^{-4}, 10^{-5}\right\}$ are displayed here. Top: initial profiles; middle: the solutions at time $t=165$; bottom: the solutions at $t=190$. See text for further details.

the gap between the two droplets, see Figure 5.3, pushes surfactant away from the near-contact region, thus generating a Marangoni stress that affects the dropletdroplet interaction. In addition, the reduction of interfacial tension due to the presence of surfactant has an effect on droplet deformation. In this way it affects the droplet-droplet interaction also as a secondary effect. 


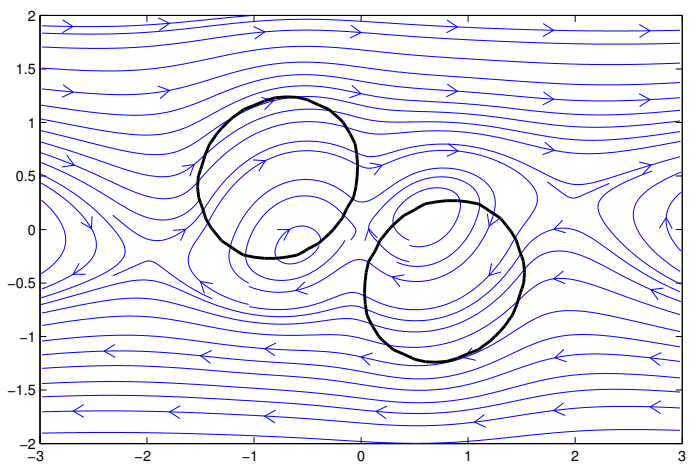

Figure 5.2. Complex flow (streamlines) for the two approaching droplets at $t=75\left(\psi_{b}=10^{-3}\right)$.

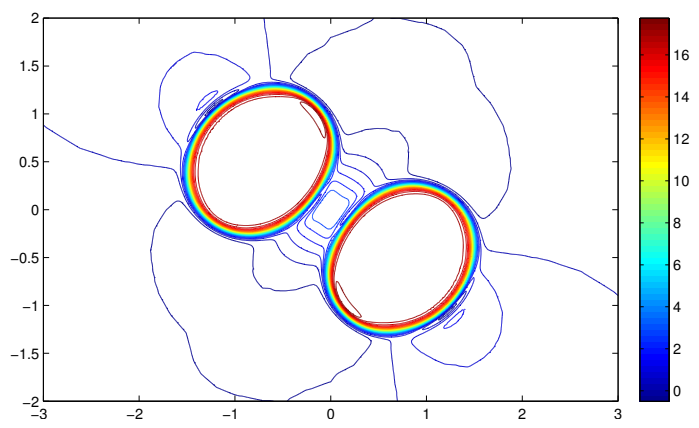

Figure 5.3. Pressure contour lines corresponding to the case in Figure 5.2.

\section{Summary AND CONClusions}

In [44], a diffuse interface model for the surfactant adsorption onto the interface of two immiscible fluids was presented. Recently, this model was extended to account also for different solubility in the two phases, and the possibility to consider systems better described by a Frumkin adsorption in addition to the Langmuir adsorption [36]. However, the basic model was the same.

Under the arguably quite weak assumption of a sufficiently smooth equilibrium solution we have shown that this model is ill-posed in the sense of frozen coefficients for a large set of physically relevant parameters, in particular for cases where the interface becomes saturated with surfactant. We have derived an explicit condition for this ill-posedness, and through careful and very accurate one-dimensional simulations illustrated that this condition is quite sharp as indicated by instability and blow-up of numerical solutions. These conclusions limit the usability of this model.

We have suggested and analyzed three alternatives to the basic model (Model 0 ), as denoted Model 1-3. In Model 1, a natural idea in the form of an energy contribution from the gradient of the surfactant concentration is included. However, besides from being more complicated, this model cannot reproduce the Langmuir adsorption isotherm, and is also found to be numerically unstable in a few cases. In Models 2-3, the surface energy accounting for the adsorption of surfactant to the 


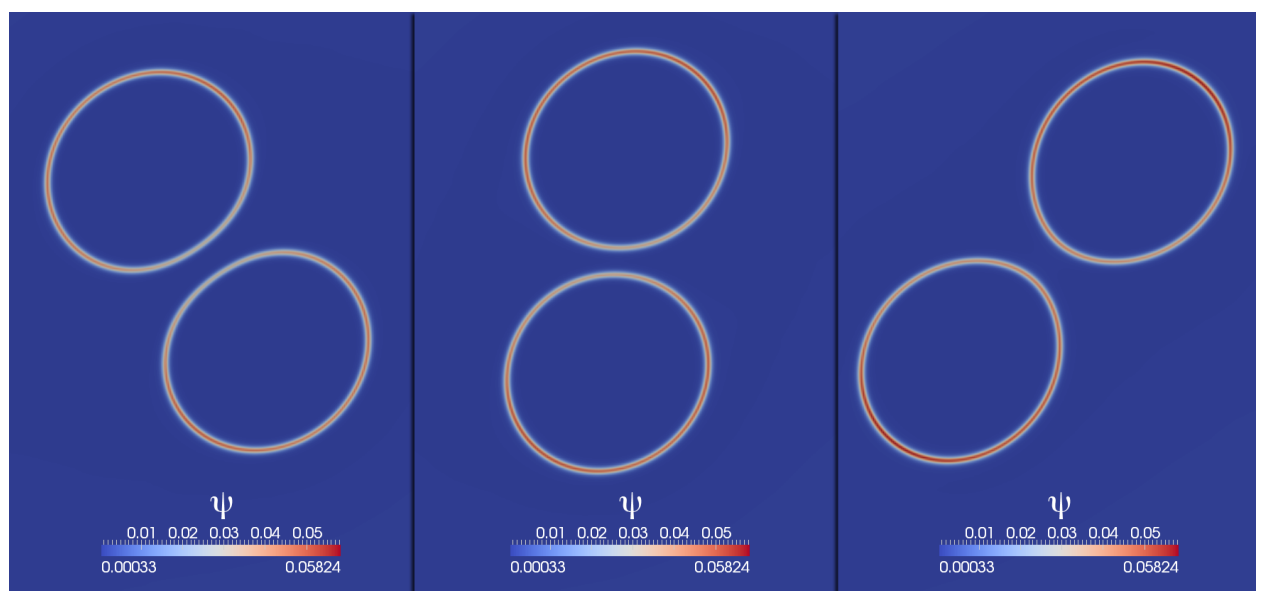

FiguRE 5.4. Surfactant concentration on the interface of the two droplets in linear shear flow. Left: $t=100$; middle: $t=125$; right: $t=150\left(\psi_{b}=10^{-3}\right)$.

interface, is changed from a form containing the gradient of the phase-field function, to a "gradient-free form". This removes the problem of ill-posedness completely, and both models are able to accurately reproduce the Langmuir adsorption isotherm. Moreover, modeling different solubility in the two phases or supporting the more general Frumkin isotherm would be straightforward [36]. The exact form of the surface energy differs between Models 2 and 3, rendering a somewhat different profile for the surfactant concentration.

The conclusions concerning the mathematical models are also verified in simulations with fluid flow. Interaction of droplets in two dimensional shear flow in presence of surfactants is taken as a test case. We find that the criteria for wellposedness determine the usability of the model also in these more complex cases.

The phase-field modeling for surfactant laden flows is still in its infancy. In this paper, we have highlighted the fact that mathematical well-posedness of the resulting equations is not an obvious feature, and that this is an issue that needs to be considered as new models are developed.

6.1. Reproducibility. Our 1D spectral-Galerkin code as described in Appendix B is available for download at the corresponding author's web-page ${ }^{1}$. Along with it, scripts that repeat the numerical experiments in Section 4 and B.3 are distributed.

\section{ACKNOWLEDGMent}

SE likes to acknowledge suggestions and comments by Per Lötstedt, Gustaf Söderlind, Bertil Gustafsson, Sara Zahedi, and Jan Hesthaven.

This work was supported by the Swedish Research Council within the FLOW and the UPMARC Linnaeus centers of Excellence. Computer time provided by SNIC (Swedish National Infrastructure for Computing) is gratefully acknowledged.

\footnotetext{
$1_{\text {http://user.it.uu.se/ stefane/freeware }}$
} 
A-KT is a Royal Swedish Academy of Sciences Research Fellow supported by a grant from the Knut and Alice Wallenberg Foundation and thankfully acknowledges this support.

Author contributions. SE wrote, developed the theory, and performed the experiments in Section 1, 3-4, and Appendix B. Section 2 and Appendix A were written by SE with inputs from MD-Q. MD-Q wrote and performed the experiment in Section 5. A-KT and GA contributed to Section 1 and wrote Section 6.

\section{REFERENCES}

[1] G. Ariel, H. Diamant, and D. Andelman. Kinetics of surfactant adsorption at fluid-fluid interfaces: Surfactant mixtures. Langmuir, 15(10):3574-3581, 1999. doi:10.1021/la980774h.

[2] J. W. Barrett, J. F. Blowey, and H. Garcke. Finite element approximation of the Cahn-Hilliard equation with degenerate mobility. SIAM J. Num. Anal., 37(1):286-318, 1999. doi:10.1137/S0036142997331669.

[3] I. B. Bazhlekov, P. D. Anderson, and H. E. H. Meijer. Numerical investigation of the effect of insoluble surfactants on drop deformation and breakup in simple shear flow. J. Coll. Interface Sci., 298(1):369-394, 2006. doi:10.1016/j.jcis.2005.12.017.

[4] A. L. Bertozzi, N. Ju, and H.-W. Lu. A biharmonic-modified forward time stepping method for fourth order nonlinear diffusion equations. Discrete Contin. Dyn. Syst., 29(4):1367-1391, 2011. doi:10.3934/dcds.2011.29.1367.

[5] M. R. Booty and M. Siegel. A hybrid numerical method for interfacial fluid flow with soluble surfactant. J. Comput. Phys., 229(10):3864-3883, 2010. doi:10.1016/j.jcp.2010.01.032.

[6] M. I. M. Copetti and C. M. Elliott. Numerical analysis of the Cahn-Hilliard equation with a logarithmic free energy. Numer. Math., 63(1):39-65, 1992. doi:10.1007/BF01385847.

[7] L. Cueto-Felgueroso and J. Peraire. A time-adaptive finite volume method for the Cahn-Hilliard and Kuramoto-Sivashinsky equations. J. Comput. Phys., 227(24):9985-10017, 2008. doi:10.1016/j.jcp.2008.07.024.

[8] T. A. Davis. Algorithm 832: UMFPACK V4.3 - an unsymmetric-pattern multifrontal method. ACM Trans. Math. Software, 30(2):196-199, 2004. doi:10.1145/992200.992206.

[9] H. Diamant and D. Andelman. Kinetics of surfactant adsorption at fluid-fluid interfaces. J. Phys. Chem., 100(32):13732-13742, 1996. doi:10.1021/jp960377k.

[10] H. Diamant, G. Ariel, and D. Andelman. Kinetics of surfactant adsorption: the free energy approach. Coll. Surf. A: Physicochem. Eng. Aspects, 183-185: 259-276, 2001. doi:10.1016/S0927-7757(01)00553-2.

[11] H. Ding, P. D. M. Spelt, and C. Shu. Diffuse interface model for incompressible two-phase flows with large density ratios. J. Comput. Phys., 226(2):2078-2095, 2007. doi:10.1016/j.jcp.2007.06.028.

[12] M. Do-Quang, W. Villanueva, I. Singer-Loginova, and G. Amberg. Parallel adaptive computation of some time-dependent materials-related microstructural problems. Bull. Pol. Ac.: Tech., 55(2):229-237, 2007.

[13] C. D. Eggleton and K. J. Stebe. An adsorption-desorption-controlled surfactant on a deforming droplet. J. Coll. Interface Sci., 208(1):68-80, 1998. doi:10.1006/jcis.1998.5816. 
[14] C. M. Elliott and H. Garcke. Diffusional phase transitions in multicomponent systems with a concentration dependent mobility matrix. Phys. D, 109(3-4): 242-256, 1997. doi:10.1016/S0167-2789(97)00066-3.

[15] D. J. Eyre. An unconditionally stable one-step scheme for gradient systems. Available at http://www.math.utah.edu/ eyre/research/methods/stable.ps, 1998.

[16] D. J. Eyre. Unconditionally gradient stable time marching the Cahn-Hilliard equation. In Computational and Mathematical Models of Microstructure Evolution, volume 529, pages 39-46, Warrendale, PA, 1998. Materials Research Society.

[17] A. Greenbaum. Iterative Methods for Solving Linear Systems. Number 17 in Frontiers in Applied Mathematics. SIAM, Philadelphia, PA, 1997.

[18] J.-L. Guermond and L. Quartapelle. A projection FEM for variable density incompressible flows. J. Comput. Phys., 165(1):167-188, 2000. doi: $10.1006 /$ jcph.2000.6609.

[19] B. Gustafsson, H.-O. Kreiss, and J. Oliger. Time Dependent Problems and Difference Methods. Pure and Applied Mathematics. John Wiley \& Sons, New York, 1995.

[20] K. Gustafsson and G. Söderlind. Control strategies for the iterative solution of nonlinear equations in ODE solvers. SIAM J. Sci. Comput., 18(1):23-40, 1997. doi:10.1137/S1064827595287109.

[21] E. Hairer and G. Wanner. Solving Ordinary Differential Equations II, Stiff and Differential-Algebraic Problems. Springer, Berlin, 2nd edition, 1996.

[22] D. Halpern, O. E. Jensen, and J. B. Grotberg. A theoretical study of surfactant and liquid delivery into the lung. J. Appl. Physiol., 85(1):333-352, 1998.

[23] R. S. Hansen. The theory of diffusion controlled absorption kinetics with accompanying evaporation. J. Phys. Chem., 64(5):637-641, 1960. doi:10.1021/j100834a027.

[24] J. S. Hesthaven and R. M. Kirby. Filtering in Legendre spectral methods. Math. Comput., 77(263):1425-1452, 2008. doi:10.1090/S0025-5718-08-021108.

[25] J. S. Hesthaven, S. Gottlieb, and D. Gottlieb. Spectral Methods for TimeDependent Problems. Cambridge Monographs on Applied and Computational Mathematics. Cambridge University Press, Cambridge, 2007.

[26] B. A. Hills. An alternative view of the role(s) of surfactant and the alveolar model. J. Appl. Physiol., 87(5):1567-1583, 1999.

[27] D. Jacqmin. Calculation of two-phase Navier-Stokes flows using phase-field modeling. J. Comput. Phys., 155(1):96-127, 1999. doi:10.1006/jcph.1999.6332.

[28] A. J. James and J. Lowengrub. A surfactant-conserving volume-of-fluid method for interfacial flows with insoluble surfactant. J. Comput. Phys., 201(2):685722, 2004. doi:10.1016/j.jcp.2004.06.013.

[29] D. Kay, V. Styles, and R. Welford. Finite element approximation of a CahnHilliard-Navier-Stokes system. Interfaces Free Bound., 10(1):15-43, 2008. doi:10.4171/IFB/178.

[30] S. Khatri. A Numerical Method for Two Phase Flows with Insoluble and Soluble Surfactants. PhD thesis, Courant Institute of Mathematical Sciences, New York University, 2009. 
[31] S. Khatri and A.-K. Tornberg. A numerical method for two phase flows with insoluble surfactants. Comput. \& Fluids, 49(1):150-165, 2011. doi:10.1016/j.compfluid.2011.05.008.

[32] I. Lagzi, S. Soh, P. J. Wesson, K. P. Browne, and B. A. Grzybowski. Maze solving by chemotactic droplets. J. Am. Chem. Soc., 132(4):1198-1199, 2010. doi:10.1021/ja9076793.

[33] M.-C. Lai, Y.-H. Tseng, and H. Huang. An immersed boundary method for interfacial flows with insoluble surfactant. J. Comput. Phys., 227(15):72797293, 2008. doi:10.1016/j.jcp.2008.04.014.

[34] A. Lamura, G. Gonnella, and J. M. Yeomans. A lattice Boltzmann model of ternary fluid mixtures. Europhys. Lett. EPL, 45(3):314-320, 1999. doi:10.1209/epl/i1999-00165-4.

[35] M. Laradji, H. Guo, M. Grant, and M. J. Zuckermann. The effect of surfactants on the dynamics of phase separation. J. Phys. Condens. Matter, 4(32):67156728, 1992. doi:10.1088/0953-8984/4/32/006.

[36] H. Liu and Y. Zhang. Phase-field modeling droplet dynamics with soluble surfactants. J. Comput. Phys., 229(24):9166-9187, 2010. doi:10.1016/j.jcp.2010.08.031.

[37] E. V. L. de Mello and O. T. da Silveira Filho. Numerical study of the CahnHilliard equation in one, two and three dimensions. Phys. A, 347:429-443, 2005. doi:10.1016/j.physa.2004.08.076.

[38] H. Mohrbach. Kinetics of nonionic surfactant adsorption at a fluid-fluid interface from a micellar solution. J. Chem. Phys., 123(12):1-4, 2005. doi:10.1063/1.2036968.

[39] M. Muradoglu and G. Tryggvason. A front-tracking method for computation of interfacial flows with soluble surfactants. J. Comput. Phys., 227(4):2238-2262, 2008. doi:10.1016/j.jcp.2007.10.003.

[40] National Institute of Standards and Technology. Digital Library of Mathematical Functions, Release date 2010-05-07. URL http://dlmf .nist.gov.

[41] A. Novick-Cohen. Chapter 4: The Cahn-Hilliard equation. In C. M. Dafermos and M. Pokorný, editors, Handbook of Differential Equations: Evolutionary Equations, volume IV, pages 201-228. North-Holland, Amsterdam, 2008.

[42] Y. Pawar and K. J. Stebe. Marangoni effects on drop deformation in an extensional flow: The role of surfactant physical chemistry. i. insoluble surfactants. Phys. Fluids, 8(7):1738-1751, 1996. doi:10.1063/1.868958.

[43] J. Shen and X. Yang. Numerical approximations of Allen-Cahn and CahnHilliard equations. Discrete Contin. Dyn. Syst., 28(4):1669-1691, 2010. doi:10.3934/dcds.2010.28.1669.

[44] R. G. M. van der Sman and S. van der Graaf. Diffuse interface model of surfactant adsorption onto flat and droplet interfaces. Rheol. Acta, 46(1):3-11, 2006. doi:10.1007/s00397-005-0081-z.

[45] G. Söderlind. Digital filters in adaptive time-stepping. ACM Trans. Math. Software, 29(1):1-26, 2003. doi:10.1145/641876.641877.

[46] G. Söderlind and L. Wang. Adaptive time-stepping and computational stability. J. Comput. Appl. Math., 185(2):225-243, 2006. doi:10.1016/j.cam.2005.03.008.

[47] H. A. Stone. A simple derivation of the time-dependent convective-diffusion equation for surfactant transport along a deforming interface. Phys. Fluids A, 
2(1):111-112, 1990. doi:10.1063/1.857686.

[48] A. M. Stuart and A. R. Humphries. Model problems in numerical stability theory for initial value problems. SIAM Review, 36(2):226-257, 1994. doi:10.1137/1036054.

[49] A. M. Stuart and A. R. Humphries. Dynamic Systems and Numerical Analysis. Number 2 in Cambridge Monographs on Applied and Computational Mathematics. Cambridge University Press, Cambridge, 1998.

[50] K. E. Teigen, P. Song, J. Lowengrub, and A. Voigt. A diffuse-interface method for two-phase flows with soluble surfactants. J. Comput. Phys., 230(2):375393, 2011. doi:10.1016/j.jcp.2010.09.020.

[51] T. Teramoto and F. Yonezawa. Droplet growth dynamics in a water/oil/surfactant system. J. Coll. Interface Sci., 235(2):329-333, 2001. doi:10.1006/jcis.2000.7349.

[52] O. Theissen and G. Gompper. Lattice-Boltzmann study of spontaneous emulsification. Eur. Phys. J. B, 11(1):91-100, 1999. doi:10.1007/s100510050920.

[53] A. F. H. Ward and L. Tordai. Time-dependence of boundary tensions of solutions I. the role of diffusion in time-effects. J. Chem. Phys., 14(7):453-461, 1946. doi:10.1063/1.1724167.

[54] T. P. Witelski, A. J. Bernoff, and A. L. Bertozzi. Blowup and dissipation in a critical-case unstable thin film equation. European J. Appl. Math., 15(2): 223-256, 2004. doi:10.1017/S0956792504005418.

[55] J.-J. Xu, Z. Li, J. Lowengrub, and H. Zhao. A level-set method for interfacial flows with surfactant. J. Comput. Phys., 212(2):590-616, 2006. doi:10.1016/j.jcp.2005.07.016.

[56] X. Ye. The Legendre collocation method for the Cahn-Hilliard equation. J. Comput. Appl. Math., 150(1):87-108, 2003. doi:10.1016/S03770427(02)00566-6.

[57] J. Zhang, D. M. Eckmann, and P. S. Ayyaswamy. A front tracking method for a deformable intravascular bubble in a tube with soluble surfactant transport. J. Comput. Phys., 214(1):366-396, 2006. doi:10.1016/j.jcp.2005.09.016.

[58] S. Zhang and M. Wang. A nonconforming finite element method for the Cahn-Hilliard equation. J. Comput. Phys., 229(19):7361-7372, 2010. doi:10.1016/j.jcp.2010.06.020.

\section{Appendix A. Non-Dimensionalization}

We discuss here in some detail the non-dimensionalization of the diffuse phasefield model incorporating surfactants as originally presented in [44]. We follow the notation therein closely in what follows.

Using the same degrees of freedom $\{\phi, \psi, \mathbf{u}\}$ as in Section 2 and with the same meaning, but now with $\phi \in\left[-\phi_{0}, \phi_{0}\right]$ we have (compare (2.1)-(2.4))

$$
\begin{aligned}
\frac{\partial \phi}{\partial t}+\nabla \cdot(\phi \mathbf{u}) & =\nabla \cdot M_{\phi} \nabla \mu_{\phi}, \\
\frac{\partial \psi}{\partial t}+\nabla \cdot(\psi \mathbf{u}) & =\nabla \cdot M_{\psi} \nabla \mu_{\psi}, \\
\nabla \cdot \mathbf{u} & =0, \\
\rho\left(\frac{\partial \mathbf{u}}{\partial t}+\mathbf{u} \cdot \nabla \mathbf{u}\right) & =-\nabla P+\nabla \cdot\left(\rho \nu\left[\nabla \mathbf{u}+(\nabla \mathbf{u})^{T}\right]\right)-\phi \nabla \mu_{\phi}-\psi \nabla \mu_{\psi} .
\end{aligned}
$$


Compared to [44], in (A.3)-(A.4) we have changed the setting to incompressible flows for which $P$ enforces the incompressibility condition and the two final terms in (A.4) acts as surface tension forces [27]. The various free parameters of the model are defined as follows.

Firstly, the free energy still takes the form (2.6) but now in terms of

$$
\begin{aligned}
F_{\phi} & =-\frac{A}{2} \phi^{2}+\frac{B}{4} \phi^{4}+\frac{\kappa}{2}(\nabla \phi)^{2}, \\
F_{\psi} & =k T[\psi \log \psi+(1-\psi) \log (1-\psi)], \\
F_{1} & =-\frac{\varepsilon}{2} \psi(\nabla \phi)^{2}, \\
F_{\text {ex }} & =\frac{W}{2} \psi \phi^{2} .
\end{aligned}
$$

In the scaling of (A.5), the equilibrium planar interface at the origin is given by $\phi(x)=\phi_{0} \tanh (x / \zeta)$ with $\phi_{0}=\sqrt{A / B}, \zeta=\sqrt{2 \kappa / A}$, and with $\{A, B, \kappa\}$ the parameters of the Cahn-Hilliard model [27].

The chemical potentials are obtained through variational derivatives of the free energy,

$$
\begin{aligned}
& \mu_{\phi}=\frac{\delta F}{\delta \phi}=-A \phi+B \phi^{3}-\kappa \Delta \phi+\varepsilon \psi \Delta \phi+\varepsilon \nabla \psi \cdot \nabla \phi+W \psi \phi, \\
& \mu_{\psi}=\frac{\delta F}{\delta \psi}=k T \log \frac{\psi}{1-\psi}-\frac{\varepsilon}{2}(\nabla \phi)^{2}+\frac{W}{2} \phi^{2} .
\end{aligned}
$$

To non-dimensionalize the model, let $\{\hat{\phi}, \hat{\psi}, \hat{\mathbf{u}}\}(\hat{t}, \hat{x})$ denote the variables and coordinates as defined in the preceding paragraph. We scale time and space in such a way that $(t, x)=\left(\hat{t} u_{0} / L, \hat{x} / L\right)$ and change variables according to $\hat{\phi}(\hat{t}, \hat{x})=$ $\phi_{0} \phi(t, x), \hat{\psi}(\hat{t}, \hat{x})=\psi(t, x), \hat{\mathbf{u}}(\hat{t}, \hat{x})=u_{0} \mathbf{u}(t, x)$, and also set $\hat{\rho}=\rho_{0} \rho, \hat{\nu}=\nu_{0} \nu$. It becomes natural to define the new parameters

$$
\mathrm{Cn}=\zeta / L, \quad \mathrm{Ex}=\varepsilon /\left(W \zeta^{2}\right), \quad \mathrm{Pi}=k T /\left(A \phi_{0}^{2}\right) .
$$

Note that, as in [44], we tacitly assume that $\varepsilon=\kappa$ in order to reduce unnecessary free parameters.

The new Ginzburg-Landau energy now becomes (2.6)-(2.11), yielding the chemical potentials in (2.12)-(2.13). The new definition of the mobilities is

$$
M_{\phi}=M_{\hat{\phi}} A, \quad m_{\psi}=m_{\hat{\psi}} A \phi_{0}^{2},
$$

where we recall that a degenerate mobility $M_{\psi}=m_{\psi} \psi(1-\psi)$ is used for $\psi$ in (A.2) (conveniently, $M_{\psi}=\psi(1-\psi)$ in (2.2)). It follows that the Péclet numbers in (2.1)-(2.2) are obtained as

$$
\mathrm{Pe}_{\phi}=L u_{0} / M_{\phi}, \quad \mathrm{Pe}_{\psi}=L u_{0} / m_{\psi},
$$

and the Reynolds and Capillary numbers as usually by

$$
\mathrm{Re}=\frac{L u_{0}}{\nu_{0}}, \quad \mathrm{Ca}=\frac{\nu_{0} \rho_{0} u_{0}}{\gamma} .
$$

Using the closing relation $\gamma \propto \kappa \phi_{0}^{2} / \zeta$ for the interfacial tension $\gamma$ finally yields (2.4). 


\section{Appendix B. Numerical Method in 1D}

In this section we describe our high-resolution one-dimensional spectral scheme for the no-flow case ( $\mathbf{u}=0$ in (2.1)-(2.2)), using the original energy (2.7)-(2.11). The necessary modifications for Model 1-3 are trivial and are omitted for brevity.

A brief review of numerical methods for gradient flows in general and surfactant models in particular might now be in order. Although many papers treat numerical methods for the Cahn-Hilliard and the closely related Allen-Cahn equations, it goes without saying that references considering computational surfactant flows are much more scarce. In this respect [5] stands out where a hybrid method for surfactant two-phase flow is designed. In the setting of a sharp interface treatment and using singular perturbation analysis, an integral formulation is used in a semi-implicit time-discretization strategy.

Semi-implicit, or split-step methods otherwise have a long history for gradient flows, but is also an active area of research. Tracing their origins back to general results in [15, (unpublished)] and [16], two more recent references include [4] and [58]. In the former, general fourth order gradient flows are considered, and in the latter the Allen-Cahn and Cahn-Hilliard equations are specifically targeted.

A somewhat different design strategy is used in $[43,56]$ where the associated Ginzburg-Landau functional is modified in order to provide for numerical stability. The design therein relies on (spectral) Galerkin formulations whereas in $[4,37]$ finite differences are rather considered. A finite volume scheme for the Cahn-Hilliard equation is obtained in [7], where the efficiency benefit with time-step adaptivity is also stressed.

On balance, and driven by a need for robustness and transparency rather than for efficiency, we have chosen to design a polynomial spectral Galerkin method with a very simple but carefully controlled time-discretization strategy. We thus postpone more advanced spatial and temporal simulation techniques for another occasion. As a word in favor of this set-up, this discretization is similar to other Galerkin-based methods such that generalizations to more realistic situations are possible.

B.1. Legendre spectral Galerkin method. We consider semi-discrete weak solutions $\phi, \psi$ to $(2.1)-(2.2)(\mathbf{u}=0)$ in the space $H^{1}(\Omega) \equiv\{w ;\|w\|+\|\nabla w\|<\infty\}$ where $\Omega=[-1,1]$ and the $L^{2}(\Omega)$-inner product and induced norm are understood. Using natural (volume preserving) homogeneous Neumann boundary conditions and the auxiliary variable $\Phi$ denoting the chemical potential $\mu_{\phi}$ we get after integration by parts the variational formulation

$$
\begin{aligned}
(\chi, \Phi) & =\left(\chi,-\phi+\phi^{3}+\frac{1}{2 \mathrm{Ex}} \psi \phi\right)+\frac{\mathrm{Cn}^{2}}{2}(\nabla \chi,(1-\psi) \nabla \phi), \\
\left(\chi, \phi_{t}\right) & =-\frac{1}{\mathrm{Pe}_{\phi}}(\nabla \chi, \nabla \Phi)
\end{aligned}
$$

for all $\chi \in H^{1}(\Omega)$ and $t \in(0, T]$ assuming available initial data at $t=0$.

The variational formulation for $\psi$ is obtained in similar fashion but using explicitly the observation in (2.14). Define first

$$
\Psi=-\frac{\mathrm{Cn}^{2}}{4}(\nabla \phi)^{2}+\frac{1}{4 \mathrm{Ex}} \phi^{2} .
$$


We tacitly assume the boundary conditions $\operatorname{Pi} \partial \psi / \partial n+\psi(1-\psi) \partial \Psi / \partial n=0$ on $\partial \Omega$ and get

$$
\left(\chi, \psi_{t}\right)=-\frac{1}{\mathrm{Pe}_{\psi}}(\nabla \chi, \operatorname{Pi} \nabla \psi+\psi(1-\psi) \nabla \Psi),
$$

again for all $\chi \in H^{1}(\Omega)$ and $t \in(0, T]$. In one dimension these boundary conditions simplify to $\phi^{\prime \prime \prime}=\phi^{\prime}=\psi^{\prime}=0$ at the endpoints $x= \pm 1$.

As a discrete subspace of $H^{1}$ we consider the space of polynomials of degree $\leq N$ and use as test- and trial functions the Legendre polynomials which are orthogonal in the $L^{2}([-1,1])$-inner product [40, Chap. 18]. Expanding $\phi_{N}(t, x)=$ $\sum_{n=0}^{N} \hat{\phi}_{n}(t) P_{n}(x)$ and similarly for $\psi_{N}$ and the auxiliary variable $\Phi_{N}$ we get from the variational form (B.1)-(B.4) the semi-discrete set of equations

$$
\begin{aligned}
M \hat{\Phi}(t) & =a, \\
M \hat{\phi}^{\prime}(t) & =\alpha, \\
M \hat{\psi}^{\prime}(t) & =\beta .
\end{aligned}
$$

The mass-matrix $M$ is diagonal with entries $M_{i i}=\left\|P_{i}\right\|^{2}=2 /(2 i+1)$ for $i \in$ $\{0, \ldots, N\}$ and

$$
\begin{aligned}
a_{i} & =\left(P_{i},-\phi_{N}+\phi_{N}^{3}+\frac{1}{2 \mathrm{Ex}} \psi_{N} \phi_{N}\right)+\frac{\mathrm{Cn}^{2}}{2}\left(P_{i}^{\prime},\left(1-\psi_{N}\right) \phi_{N}^{\prime}\right), \\
\alpha_{i} & =-\frac{1}{\mathrm{Pe}_{\phi}}\left(P_{i}^{\prime}, \Phi_{N}^{\prime}\right), \\
\beta_{i} & =-\frac{1}{\mathrm{Pe}_{\psi}}\left(P_{i}^{\prime}, \operatorname{Pi} \psi_{N}^{\prime}+\psi_{N}\left(1-\psi_{N}\right) \Psi^{\prime}\right),
\end{aligned}
$$

with $\Psi$ defined by (B.3) (hence imposed strongly). The derivatives are readily obtained by explicit differentiation of the Legendre expansions [25, Appendix B.1.4].

To evaluate the inner products we use the associated Gauss-Legendre quadrature of at least the same order as the scheme, often considerably higher in order to avoid or at least mitigate aliasing errors [25, Chap. 6]. It is known that, for nonlinear operators, aliasing errors and Gibbs oscillations near sharp gradients may drive the scheme unstable even for smooth problems [24]. This problem is perhaps mainly associated with shocks but is of relevance also here since phase-field models naturally produce sharp gradients in the vicinity of the interface.

We have therefore implemented filtering along the lines presented in [25, Chap. 9.2]. To this end we replace the Legendre expansion with the filtered expansion

$$
\phi_{N}(t, x)=\sum_{n=0}^{N} \vartheta(n / N) \hat{\phi}_{n}(t) P_{n}(x),
$$

and similarly for $\psi_{N}$ and $\Phi_{N}$. A suitable filter is the exponential one defined by [25, p. 164]

$$
\vartheta(\eta)=\left\{\begin{array}{cl}
1 & \eta \leq \eta_{c}, \\
\exp \left(-\alpha\left(\frac{\eta-\eta_{c}}{1-\eta_{c}}\right)^{p}\right) & \eta>\eta_{c}
\end{array}\right.
$$

with $\left[\eta_{c}, p, \alpha\right]$ filter parameters. Following the recommendations in [24] we take the order to be $p=12$ and set $\alpha=-\log \epsilon \approx 36$ with $\epsilon$ the double precision machine accuracy. We also specify $\eta_{c}=0.25$ so that the $25 \%$ low modes are not filtered 
at all. The filtered method is then conveniently implemented by replacing the mass-matrix in (B.5)-(B.7) with $\tilde{M}$ defined by $\tilde{M}_{i i}:=\vartheta(i / N)^{-1} M_{i i}$.

B.2. Adaptive discretization of time. Since we are considering problems that either develop instabilities or for which there are nearby ill-posed problems, rather than aiming for efficiency and high order we simply use the backward Euler method for discretizing (B.5)-(B.7) in time:

$$
\begin{aligned}
\tilde{M} \hat{\Phi}^{k+1} & =a^{k+1}, \\
\tilde{M}\left(\hat{\phi}^{k+1}-\hat{\phi}^{k}\right) & =\Delta t \alpha^{k+1}, \\
\tilde{M}\left(\hat{\psi}^{k+1}-\hat{\psi}^{k}\right) & =\Delta t \beta^{k+1},
\end{aligned}
$$

with $\Delta t$ a suitable time-step and where the dependence on time is expressed through superscripts.

Although there are arguably more efficient split-step methods available that would probably apply here $[4,37,58]$, the backward Euler method has a specific advantage for gradient flows such as the one at hand. Namely, it is one of very few unconditionally gradient stable methods (if not the only one), which preserves decay in the exact energy functional of the system (cf. [49, Chap. 5.6.1]; Theorems 5.6.15.6.3). Correct energy dissipation has been recognized as one of the most important criteria when designing numerical methods targeting gradient flows, which in specific cases may display chaotic behavior and "weak turbulence" (see [48, Sect. 4] and [7]). An intuitive argument is the fact that the Ginzburg-Landau energy is the modeling step and controlling the rate of energy dissipation is therefore more important than formal order.

For the Cahn-Hilliard equation it has been demonstrated by example that timestep adaptivity is of vital importance since the dynamics is rich in scales and since rapid transients due to initial data and boundary conditions occur naturally [7, 37]. We address this by using error estimates from half-steps and a careful time-step selection mechanism.

Modern adaptivity based on digital filtering was used in our code [45, 46]. To discuss it and for brevity, let $T_{\Delta t}$ be the forward-in-time map by the backward Euler method as obtained by solving (B.13)-(B.15). We put $u_{1}=T_{\Delta t} u_{0}$ and $u_{2}=T_{\Delta t / 2}^{2} u_{0}$ for some initial state $u_{0}$. By Richardson extrapolation this gives us a point-wise error estimate

$$
\text { err }=\left|u_{1}-u_{2}\right| / 3 .
$$

For given relative and absolute tolerances Rtol and Atol we compare the error to the effective tolerance vector TOL $:=\max \left(\mathrm{Rtol}\left|u_{2}\right|\right.$, Atol $)$ through the control variable

$$
\text { cerr }:=L\left(\| \text { err } / \text { TOL } \|_{\infty}^{-1 / 2}\right),
$$

(element-wise division), where $1 / 2$ is the order of the error estimate and where $L$ is a denoising limiter (see below). We consistently used the fairly stringent tolerances Rtol $=10^{-6} \times \operatorname{diag}\left(M^{-1 / 2}\right)$ (vector) and Atol $=10^{-8}$ in our experiments.

The control objective is now to keep cerr $=1$. An updated step-size is obtained from a previous time-step $\Delta t^{\prime}$ via the relation $\Delta t=\rho \Delta t^{\prime}$, where in terms of

$$
\rho:=L\left(F\left(\operatorname{cerr}, \operatorname{cerr}^{\prime}, \rho^{\prime}\right)\right)
$$


and where primes denote variables computed at the previous time-step. As to the choice of $F$, the step-size controller, we decided upon H211b defined by

$$
F(\alpha, \beta, \gamma)=\alpha^{1 / b} \beta^{1 / b} \gamma^{1 / b}
$$

with the recommended choice of the single parameter being $b=1 / 4$ [45]. Both (B.17) and (B.18) employ the arctan-limiter [46, Sect. 6]

$$
L(\alpha)=1+\kappa \arctan \left(\frac{\alpha-1}{\kappa}\right),
$$

with, respectively, $\kappa=2$ in (B.17) and $\kappa=1$ in (B.18). The limiter's purpose in the latter case is to smoothly restrict the rate by which the step-size may change and, in the former case, to reduce the impact of noise in the error estimate.

By the very action of the step-size controller, the sequence of $\rho \mathrm{s}$ is generally much smoother than the corresponding values for the control variable in (B.17). For computational stability and following a suggestion in [46], we therefore base the decision of rejecting steps on the former variable rather than on the latter. Specifically, given some tolerance factor $M$ (we took $M=2$ ), the controller is scheduled to reject the step whenever err $>M \cdot T O L$. Following the steps that lead to (B.18) this suggests the rejection criterion

$$
\rho<\rho_{\min }:=L\left(F\left(L\left(M^{-1 / 2}\right), 1,1\right)\right) \approx 0.9179 .
$$

In practice and with this strategy for time-step selection, step rejections occur very rarely.

For solving the nonlinear system of equations (B.13)-(B.15), simplified Newton iterations with a numerical Jacobian were used. We followed the prescription for estimating the speed of convergence in [21, Chap. IV.8], but also found it rewarding to augment the code with control strategies devised in [20] for reusing previous Jacobian evaluations and factorizations.

The above described code was used successfully over a wide range of test-cases. For reproducibility, the Matlab source code is made freely available from the corresponding author's web-page. In particular, scripts generating all numerical results in Section 4 are distributed (see Section 6.1 for further information).

B.3. Convergence. In order to assess the convergence properties of the code we performed simulations for the pure Cahn-Hilliard part with $\mathrm{Pe}_{\phi}=1, \mathrm{Cn}=0.1$, $u=0.25$, and time $t \in[-0.5,0.5]$. In this case, due to the non-zero flow $u$, there is an additional term $(\chi, u \nabla \phi)$ in (B.2). The reason for adding transport is that for the corresponding free-space formulation there is a traveling wave solution $\phi(t, x)=$ $\tanh (x-u t)$, which in the stated interval of time is an accurate solution also for the present case with homogeneous Neumann boundary conditions. We parametrize the tolerances for the time discretization according to Rtol $=\varepsilon \times \operatorname{diag}\left(M^{-1 / 2}\right)$ and Atol $=\varepsilon \times 10^{-2}$, with $\varepsilon$ a single parameter.

To test also the surfactant formulation (2.1)-(2.2), a highly accurate $(N=400$, $\varepsilon=10^{-10}$ ) reference solution was computed for the purpose of estimating errors. In this case a constant surfactant profile $\psi=10^{-3}$ was used as the initial data, and surfactant parameters $\mathrm{Pe}_{\psi}=1, \mathrm{Ex}=1, \mathrm{Pi}=0.1$ for Model 3 were settled upon. The rest of the model was kept identical with the previous case of pure Cahn-Hilliard flow.

The results of these experiments are shown in Figure B.1. The coupled problem is evidently a more difficult one, partially due to aliasing errors in this more strongly 


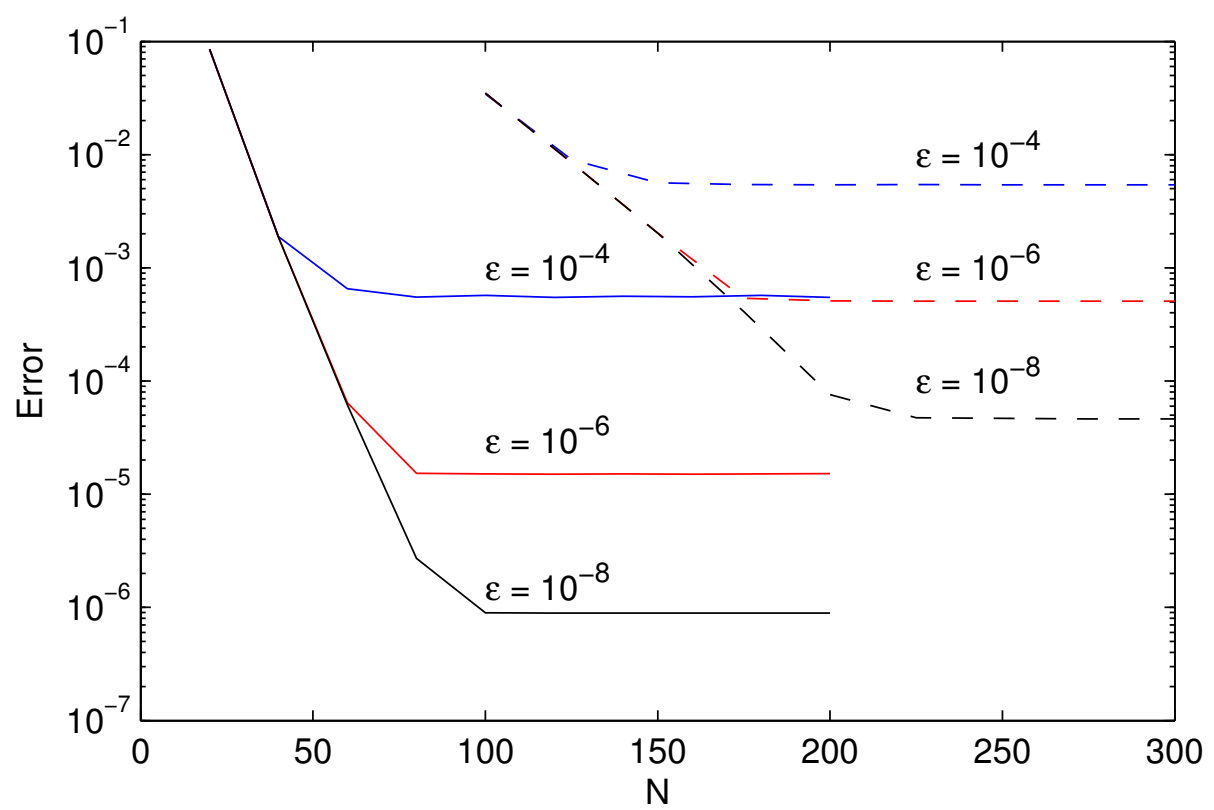

Figure B.1. Convergence study for the 1D code (relative error in the $L^{2}([-1,1])$-norm, maximum over the interval of integration). Three different time-stepping tolerances $\varepsilon$ and increasing order $N$ of the polynomial basis. Solid: traveling wave solution (pure CahnHilliard case), dashed: coupled case, comparision with high order reference solution.

nonlinear formulation, but also due to the relative sharpness of the surfactant profile. In all cases spectral convergence down to a level dictated by the accuracy of the time discretization are clearly visible.

(S. Engblom) Division of Scientific Computing, Department of Information Technology, Uppsala University, SE-751 05 Uppsala, Sweden.

$U R L$, S. Engblom: http://user.it.uu.se/ stefane

E-mail address: stefane@it.uu.se

(M. Do-Quang and G. Amberg) Linné Flow Centre, Department of Mechanics, School of Engineering Science, Royal Institute of Technology, S-100 44 Stockholm, Sweden

E-mail address: minh@mech.kth.se, gustava@mech.kth.se

(A-K. Tornberg) Linné Flow Centre, Department of Numerical Analysis, School of Computer Science and Communication, Royal Institute of Technology, S-100 44 StockHOLM, SwEDEN

E-mail address: annak@nada.kth.se 